Aus dem Anatomischen Institut der Tierärztlichen Hochschule in Dresden. (Prof. Dr. B a um.)

\title{
Vergleichende Untersuchungen über den mikroskopischen Bau der Lymphdrüsen von Pferd, Rind, Schwein und Hund. \\ Von \\ Johannes Richter.
}

Hierzu Tafel XXIV und XXV.

Die in das Lymphgefasssystem eingeschalteten Lmyphknoten oder Lymphdrüsen sind, wie die reiche Litteratur beweist, häufig Gegenstand makroskopischer und mikroskopischer Untersuchungen gewesen. Hierbei dürften die Lymphdrüsen unserer Haussäugetiere zum Teil wohl nur deshalb in den Rahmen der Forschung hereingezogen worden sein, weil die an und in ihnen gefundenen Verhältnisse ein Mittel für den Zweck der Klarlegung des Baues der menschlichen Lymphknoten bilden sollten. Und so kann es nicht Wunder nehmen, dass trotz der zahlreichen und von den verschiedensten Seiten vorgenommenen Untersuchungen die Kenntnisse über die Lymphdrüsen der Tiere immer noch bedeutende Lücken aufweisen, und zwar namentlich deshalb, weil in der Regel nur die bequem aufzufindenden und besonders grossen Lymphdrüsen bei den Untersuchungen bevorzugt worden sind. Die durch die Betrachtung einzelner Drüsen erzielten Ergebnisse sind dann stillschweigend als für die Gesamtheit der Lymphdrüsen gültig angenommen worden. Hierbei macht sich der Mangel eines alle Lymphdrüsengebiete gleichmässig umfassenden Vorgehens deutlich fühlbar. Diese Thatsachen veranlassten Herrn Prof. Dr. Baum, Professor der Anatomie an der Königlichen Tierärztlichen Hochschule zu Dresden und Vorstand des anatomischen Instituts, mich mit der vergleichend-histologischen Untersuchung der Lymphdrüsen aller Körperregionen von Pferd, Rind, Schwein und Hund zu betrauen. Diese Arbeit wurde von mir in Leipzig begonnen und im anatomischen Institut der Tierärztlichen Hochschule in Dresden fortgesetzt und beendet. Für Ueberlassung des Themas, sowie für die liebenswürdige Anregung und wissenschaftliche Förderung gestatte ich mir, Herrn Prof. Dr. Baum, meinem hochverehrten Lehrer, meinen herzlichen, tiefempfundenen Dank auch an dieser Stelle auszusprechen. 
Es handelt sich in nachstehender Arbeit um Beantwortung der Fragen:

1. Bestehen histolqgische Unterschiede zwischen den verschiedenen Stellen des Körpers entnommenen Lymphdrüsen von Tieren derselben Gattung, und welcher Art sind diese Unterschiede?

2. Wodurch unterscheiden sich die Lymphdrüsen der Vertreter von verschiedenen Tiergattungen - Pferd, Rind, Schwein, Hund - in Bezug auf ihren histologischen Bau?

G. Eckard (1) konnte Strukturunterschiede zwischen den äusserlichen und den im Innern des Körpers liegenden Drüsen nicht finden. Die spăteren Autoren stehen in der Hauptsache auf einem anderen Standpunkte. So schreibt z. B. F. v. Recklingha isen (2): „Die Lymphdrüsen zeigen sowohl bei den verschiedenen Tiergattungen, wie auch in einem und demselben Individuum einen verschiedenen, anscheinend schwer zu definierenden Bau." In ähnlichem Sinne sprechen sich $\mathrm{His}(3)$, Teichmann (4), Kölliker (5 und 6), Frey (7) u. a. aus, deren Ausführungen zum Teil noch heranzuziehen sein werden. In den bișher erschienenen einschlägigen Abhandlungen konnten nur Mitteilungen gefunden werden, welche über einzelne bestimmte Lymphdrüsen vom Standpunkte der Histologie gemacht sind. Diese Mitteilungen beschrănken sich fast ausschliesslich a u Befunde in den Axillar-, Inguinal-und Mesenterialdrüsen von Rind und $H$ u n ; nebensächlicher werden einzelne Lymphdrüsen von Pferd, Schwein und anderen Tieren betrachtet.

Die von $\mathrm{mir}$ angestellten Untersuchungen haben dagegen in erster Linie den Zweck, alle Lymphknotengebiete des Körpers von Pferd, Rind, Schwein und Hund gleichmässig zu berücksichtigen. Die Wahl der zu untersuchenden Lymphknoten wurde im wesentlichen bestimmt durch die in der vergleichenden Anatomie von Ellenberger und Ba m (8), sowie in der Anatomie des Hundes derselben Autoren (9) befindliche Einteilung der Glandulae lymphaticae, sodass folgende Drüsen in Betracht kommen, von denen für den Hund nur die mit * versehenen in dem betreffenden Abschnitt berücksichtigt werden können: 
Vergleichende Untersuchungen über den mikroskopischen Bau etc. 471

1. *submaxillare Lymphknoten, Lymphoglandulae submaxillares,

2. *retropharyngeale Lymphknoten, Lg. retropharyngeales,

3. *obere, Lg. cervicales craniales,

4. *mittlere, Lg. cervicales mediae,

5. *untere Halslymphdrüsen, Lg. cervicales caudales,

6. *Bug-, Lg. cervicales superficiales,

7. Ellenbogen-, Lg. cubitales (nur beim Pferd vorhanden),

8. *Achsel-, Lg. axillares,

9. *Kniekehlen-, Lg. popliteae,

10. *Kniefalten-, Lg. subiliacae externae,

11. *Scham-, Lg. inguinales superficiales,

12. Leistendrüsen, $\mathrm{Lg}$. inguinales profundae,

13. Lymphknoten der Brustwand, Lg. thoracis,

14. Mittelfell-, Lg. mediastinales,

15. *Bronchial-, Lg. bronchiales,

16. * Lenden-, Lg. lumbales,

17. *Nierendrüsen, Lg. renales,

18. *mediale Darmbein-, Lg. iliacae internae,

19. Iaterale Darmbeindrüsen, Lg. iliacae externae,

20. *Lymphknoten der Leber, Lg. hepaticae,

21. der Milz, Lg. lienales,

22. *des Magens, Lg. gastricae,

23. *des Gekröses, Lg. mesentericae, mit denen

24. des Dünndarmes und

25. *des Dickdarmes.

Die von frisch getöteten Tieren stammenden Drüsen wurden in Alkohol gehärtet - die kleineren in ihrer Totalität, die grösseren in Scheiben zerlegt - , in Paraffin eingebettet, nach der von Le e und M a y r (10) angegebenen Methode mit reinem Wasser auf Objektträger aufgeklebt, in "stark verdünnter Hämatoxylin- und Eosinlösung gefärbt und nach der üblichen Behandlung mit Alkohol und Xylol in Canadabalsam eingeschlossen. Sämtliche Schnitte gestatten somit Einblick in die natürlichen Verhältnisse, soweit dies bei präparierten Objekten möglich ist.

Pferd.

$\mathrm{Zu}$ den mikroskopischen Untersuchungen über die Lymphdrüsen des Pferdes wurde das Material aus den 25 verschiedenen Drüsengebieten von drei ganzen Tieren verwendet, sowie eine 
grössere Anzahl von Schnitten. Diese waren von mir aus Lymphknoten angefertigt, welche gleichfalls aus den 25 Regionen allerdings verschiedener Pferde stammten. Aus dem Studium der mikroskopischen Präparate hat sich für den Bau der Lymphdrüsen des Pferdes folgendes ergeben.

\section{Gerüstsubstanz.}

Die Hülle und das grobe Stützgerüst der Pferdelymphdrüsen bestehen wie bei allen Lymphdrüsen aus fibrillärem Bindegewebe und eingestreuten glatten Muskelfasern. In verschiedenen Drüsen finden sich in diesen Bindegewebszügen Maschen von ca. $8 "$ Weite, wie schon Sussdorf (11) angiebt; in diese Maschen sind Lymphzellen eingelagert. Das Septensystem ist beim Pferde sehr schwach in der Rindensubstanz entwickelt, stårker tritt es in der Marksubstanz auf. Ganz besonders hervorgehoben $z u$ werden verdient, dass die Dicke der Hülle und die Stärke der Trabekeln bei den einzelnen Lymphdrüsengruppen bedeutenden Schwankungen unterworfen sind. Auf diese Thatsache hat zwar im allgemeinen schon Frey (12) aufmerksam gemacht; denn er schreibt: "Zahlreiche Beobachtungen bei verschiedenen Säugetieren haben nun zwar viele untergeordnete Variationen, aber keine wesentlichen Verschiedenheiten ergeben. Die Kapsel erscheint bald starker, bald feiner." Weiter sagt er: „Die Machtigkeit der Scheidewande wechselt indessen sehr. So sind bei den Gekrösdrüsen mancher Săugetiere die Scheidewände gleich den Kapseln auffallend feiner als $z$. B. bei den mehr äusserlich gelegenen Lymphdrüsen." Durch meine Untersuchungen können diese Angaben zum Teil bestatigt, vor allem aber erheblich erweitert werden. Eine statistische Zusammenstellung hat nämlich betreffs der Zunahme der Stärke der Hülle und der Trabekeln eine gewisse Steigerung für die verschiedenen Lymphdrüsen jedes einzelnen Individuums derart ergeben, dass man die Lymphknoten, ungefähr wie folgt, ordnen kann. Zu der Gruppe, bei welcher Kapsel und Septen am wenigsten entwickelt sind, gehören die Leber- und Milzdrüsen, diejenigen des Magens und Darmkanals, sowie die Bronchial- und Mediastinaldrüsen. Gerüstreicher sind gewöhnlich schon die Leniden- und Nierendrüsen, und dann folgen im Verhältnis der Zunahme der Gerüst- 
substanz etwa die inneren und äusseren Darmbeindrüsen, ferner die Lymphknoten des Kehlkopfs, der Brustwand und die Submaxillardrüsen.. Ihre stärkste Entwicklung finden Kapsel und Septen in der Regel bei den Bug-, Scham- und Leistendrüsen, den Lymphknoten des Halses und endlich den Kniekehlen-, Ellenbogen-, Kniefalten- und Achseldrüsen.

Bezüglich der Kapsel lässt sich Bemerkenswertes weiter nicht sagen, aber: in Hinsicht auf die Trabekeln sei noch folgendes hinzugefügt. Die von der Kapsel abzweigenden Septen sind nämlich bei den dem Darmkanal und seinen Anhängen zugehörigen Drüsen, sowie den Bronchial- und Mediastinaldrüsen auf Normalschnitten entweder nicht oder nur vereinzelt $\mathrm{zu}$ sehen, - die Kapsel zeigt bisweilen nur die Andeutung einer Scheidewand durch eine geringe Verdickung -, oder die Septen sind sehr schwach und schmal, während in Schnitten der Inguinal-, Halsund anderen Muskellymphdrüsen häufig von der Kapsel breite Septen die ganze Drüse durchsetzen oder wenigstens durch starke Querschnitte als solche sich zu erkennen geben. Diese beiden Gruppen stehen sich direkt gegenüber; die Brücke zwischen ihnen schlagen die den Wandungen der beiden grossen Körperhöhlen anliegenden Lymphknoten. Dieses Verhăltnis zeigt sich auch bei ălteren Tieren ausgeprägt; es kommt bei diesen vielleicht eher noch schärfer dadurch zum Ausdruck, dass die an sich gerüstreichen Organe der äusseren Drüsenregionen raṣcher der senilen Bindegewebsneubildung anheimfallen. Wenigstens scheint diese Annahme auf Grund der Präparate, die von einem ungefähr 25jährigen Pferde stammen, berechtigt zu sein.

Im engsten Zusammenhange mit dem Gerüstwerk steht das Hilusstroma, jedoch machen die in dessen Auftreten vorkommenden Schwankungen eine eingehendere Erörterung notwendig. Es ist schon verschiedentlich darauf hingewiesen worden, dass ein Hilus durchaus nicht allen Drüsen eigen ist. Sus sdorf schreibt z. B.: „Ein eigentliches Hilusstroma, wie das allgemeine Schema der Drüsen ein solches lehrt, findet sich durchaus nicht bei allen Tieren gleichmässig entwickelt vor." An der Hand der von mir angefertigten Präparate konnte nun festgestellt werden, dass nur bei ca. $60 \%$ der Lymphdrüsen des Pferdes ein Hilus vorhanden ist; bei ungefähr $40 \%$ derselben war die Einsenkung des Hilusstromas deutlich zu er- 
kennen (cf. Fig. 1, h.), d. h. die Gestalt der Drüsen năherte sich dadurch der der Nieren; bei $20 \%$ war die Einsenkung weniger scharf ausgeprăgt und der Hilus war mehr durch eine die Gefässe führende Verstärkung der Kapsel angedeutet. Bei den übrigen Drüsen war nur an einer der schwach konvexen Seiten der Ein- bezw. Austritt der Blut- bezw. Lymphgefässe zu erkennen. Diese Variationen erstreken sich nach meinen Untersuchungen gleichmassig auf alle Lymphdrüsengebiete, sodass eine Einteilung nach dem Vorhandensein oder Fehlen des Hilus nicht aufgestellt werden kann.

Auch die Entwickelung der bindegewebigen Masse des Hilus, falls ein solcher eben da ist, ist bei den einzelnen Lymphdrüsen verschieden, worauf schon Frey aufmerksam macht; denn er schreibt in seinem Handbuch: „Dieselbe zeigt die allerbeträchtlichsten Schwankungen. Während sie nämlich an manchen innern Lymphknoten nur höchst unbedeutend getroffen oder geradezu vermisst wird, kann sie an anderen, namentlich ăusserlich gelegenen, eine gewaltige Măchtigkeit erlangen, sodass sie das lymphoide Gewebe der Marksubstanz zu verdrängen beginnt." Die Befunde von Frey werden durch meine Untersuchungen in der Hauptsache bestätigt, nur. muss ich hervorheben, dass, wie aus dem oben Gesagten hervorgeht, das Hilusstroma a u ch äusserlich gelegenen Drüsen fehlenkann. Dass es aber bei diesen an Stărke demjenigen der im Innern befindlichen Drüsen häufig überlegen ist, hängt einfach damit zusammen, dass eben im allgemeinen bei ersteren Hülle und Gerüst reichlicher ausgebildet sind. Hier dient das Hilusstroma vorzüglich als Gerüstsubstanz, indem es oft hoch in die Drüse aufsteigt und sich weit verzweigt, wobei scharf begrenzte, zum Teil abgeschlossene Råume und Spalten gebildet werden, welche die Lymphoidmassen in sich aufnehmen. (cf. Fig. 2, hs u. $\mathrm{r}^{1}$ ). Es ergänzt also das Hilusstroma in diesen Lymphdrüsen das von der Kapsel abgehende Septensystem in den zentralen und in den in der Năhe des Hilus gelegenen Partien namentlich jener Drüsen, die über ein stärkeres Gerüst verfügen. Bei einigen Lymphknoten trennt sogar das Hilusgewebe die Drüsensubstanz in zwei Teile, die dann zu beiden Seiten des gefăssreichen Stranges liegen, indem es das Parenchym vollkommen durchdringend an die Oberfläche tritt 
Vergleichende Unterșuchụngen über den mikroskopischen Bau etc. 475

und sich hier beiderseits in die Kapsel fortsetzt, was bei Lymphdrüsen ohne Hilusstroma natürlich unmöglich ist.

\section{Parenchym.}

Die lymphoiden Gebilde der Drüsen, welche bekanntermassen von der Gerüstsubstanz durch die Lymphwege getrennt sind, haben zuerst durch Brücke (13) eine Scheidung in Rind.e und M ark erfahren. Indem er zugleich Bezug nimmt auf die verschiedene Verteilung beider Substanzen, schreibt er in der Denkschrift: „Die Marksubstanz besitzt relativ zur Rindenschicht eine sehr verschiedene Ausdehnung je nach der Spezies und dem Alter, vielleicht auch je nach dem aktuellen Zustande des Individuums. Bei Menschen, Hunden und Schafen ist sie verhälnnismässig bedeutend." Dass eine derartig strenge Scheidung in Rinden- und Markschicht den Verhaltnissen durchaus nicht immer entspricht, ist hinlanglich bekannt, da sich eine „Schichtung" nur in seltenen Fällen nachweisen lässt. Doch ist es aus Gründen der Zweckmässigkeit nnd der besseren Verständigung nicht zu umgehen, von Rinden- und Mark-,,substanz"6 zu sprechen. Das Verhältnis beider zu einander ist nun beim Pferd ein denkbar verschiedenes. Das ist das Hauptergebnis der Untersuchungen meiner Schnitte. In manchen Drüsen findet man ein breit zusammenhängendes lymphoides Gewebe, welches fast nicht einmal Andeutungen von Lymphsträngen erkennen lässt, und in völligem Gegensatz hierzu stehen andere Drüsen, welche nur aus Marksubstanz zu bestehen scheinen, und zwar treten solche Bilder auch in den zur Längsachse der Drüse senkrecht gelegten Schnitten auf, nicht nur in Flachschnitten, bei denen dieses Verhältnis leichter denkbar ist. Zwischen den beiden Extremen liegt die grosse Masse der Fälle, wo beide Substanzen zu erkennen sind.

\section{a. Rindensubstanz.}

Bei der überwiegenden Zahl der Lymphdrüsen des Pferdes findet man eine wohl ausgeprägte Rindensubstanz. Sie nimmt im allgemeinen mehr die peripheren Regionen des Lymphknotens ein; doch kommen viele Abweichungen von diesem Schema vor, auf welche bei Besprechung der Marksubstanz noch näher eingegangen werden soll, weil sich an jener Stelle die 
Klarlegung dieser Verhältnisse übersichtlicher gestalten dürfte. Hier sei nur in Bezug auf die Stärke der Rinde erwähnt, dasss das ihr zukommende zusammenhängende Gewebe nach meinen Untersuchungen seine höchste Ausbildung bei den Bronchial-, Mediastinal- und Magenlymphdrüsen zeigt, dagegen am wenigsten bei den Lymphdrüsen des Darmkanals entwickelt ist, wo man häufig nur einen schmalen Saum antrifft, der auch noch Unterbrechungen durch Markstränge und Lymphwege aufweisen kann. In der Mitte stehen die anderen Drüsen. Im übrigen lehrt eine einfache Ueberlegung, dass bei bekanıter Ausdehnung der Marksubstanz die Stärke der Rinde im umgekehrten Verhältnis zu jener stehen muss. Selbstverständlich kann man diesen Satz nicht mit mathematischer Genauigkeit durchgeführt finden und die Strukturverhaltnisse lebender Organe nicht in solche starle Formen bringen wollen, aber ein Hauptgesichtspunkt bei der Erklärung über die Verteilung von Rinde und Mark ist dadurch doch gegeben. - Entsprechend der geringen Ausbildung des Septensystems treten beim Pferde die Rindenpartien dem Beobachter in der Hauptsache als breit zusammenhängende lymphoide Massen entgegen, in die nur hier und da ein Septum von der Kapsel niedersteigt oder in denen einige Querschnitte von solchen zu treffen sind. Hierbei fällt natürlich vor allem ins Gewicht; aus welcher Lymphknotengruppe die betreffende Drüse stammt, indem die gerüstreicheren Lymphknoten eine Trennung in Unterabteilungen (,Follikel", „Ampullen" der alteren Autoren, „,Sekundärknötchen“" Stöhr [14]) in reicherem Masse erleiden müssen als die gerüstarmen. Hieraus folgt, dass die Sekundarknotenbildung') bei den Kniefalten- oder Achseldrüsen deutlicher ins Auge fallen wird als z. B. bei den Darmdrüsen.

Einer besonderen Besprechung bedürfen die der Rindensubstanz eigentümlichen Sekundärknötchen (Flemming [15]). Dieselben wurden das erste $\mathrm{Mal}$ von $\mathrm{His}$ erwähnt und als "Vakuolen" bezeichnet. His behandelt diese ,kugelrunden Hohlräume" ziemlich eingehend und giebt bezüglich ihrer Lage bei einigen Rinderdrüsen an: ,eigentümlich ist auch die exzentrische Lagerung derselben; sie finden sich nicht in der

1) Um Missverständnisse zu vermeiden, sei hier bemerkt, dass in dieser Arbeit für die unzutreffende Bezeichnung „Follikel“ ${ }^{4}$ die Benennung "Sekundärknoten" gewählt wurde. 
Mitte, sondern in der Regel ganz am Rand der Alveolen gelagert, in der Nähe der Septa oder: Drüsenhülle, von diesen nur durch einen: schmalen Streif zwischenliegender Drüsenisubstanz und den Lymphsinus geschieden." Diesen „Vakuolen" ist weiterhin wenig Aufmerksamkeit geschenkt worden. So scheidet z. B. Sussdorf die ,Follikel" überhaupt nicht von den Sekundărknoten, und Teichmann schreibt nur: „Die His'schen Vakuolen habe ich nicht gesehen." Krause (16), Kölliker und Armauer-Hansen (17) hingegen bestätigen die Angaben von His. Aber erst Flemming hat über den Charakter und die Funktion dieser Gebilde Aufklärung geschaffen. Er hat bewiesen, dass sie die Hauptbildungsstătten der Lymphzellen in den Lymphknoten sind, und hat sie morphologisch als ,Sekundäknötchen", physiologisch als „Keimzentren" benannt. Diese letztere Bezeichnung soll hier beibehalten werden. Die Keimzentren sind bis jetzt nur beim Rind und Hund ( $\mathrm{His}$, Krause, Kölliker Armauer-Hansen) und beim Schwein (Chievitz [18]) beschrieben; sie finden sich nach meinen Untersuchungen jedoch in den weitaus meisten Fällen auch beim Pferd, und zwar zählt man durchschnittlich im Gesichtsfeld willkürlich eingestellter Drüsenschnitte bei schwacher Vergrösserung ( $\mathrm{Zeiss}$, Okular 2, Objektiv A) 2,56 Keimzentren, die im Mittel $0,245 \mathrm{~mm}$ messen.

Die oben angeführte, das Rind betreffende Lagebeschreibung der Keimzentren von $\mathrm{H}$ is kann, soweit sich aus meinen Präparaten ersehen lässt, als für das Pferd gültig bezeichnet werden, wenn man für dieses Tier einfügt ,die Mehrzahl der Keimzentren.“ Es liegen mithin beim Pferd die Keimzentren in der Rindensubstanz und zwar am dichtesten in der unmittelbaren Nähe der Drüsenoberfläche sowie der Septen, weniger zahlreich in den zentralen Partien der Rindensubstanz. Letzterer Fall tritt am häufigsten bei den gerüstarmen Drüsen auf, die gleichzeitig eine gering entwickelte Marksubstanz besitzen, das sind die Bronchial-, Mediastinal- und Magendrüsen. Hiermit ist aber nicht gesagt, dass diesen genannten Drüsen etwa die Höchstzahl der Keimzentren zukäme. Es unterliegt vielmehr deren Zahl in allen Drüsen erheblichen Schwankungen. In etwa $10 \%$ aller Lymphknoten waren z. B. Keimzentren überhaupt nicht nachweisbar. Auf diese Thatsache, dass man in 
manchen Drüsen vergeblich nach den Keimżentren suchèn katih; haben Armater-Hansen und Flemming: aufmerksam' gemacht. Ersterer giebt an, , ,dass man in' vielen' Drüseri; besonders solchen ohne eigentliche Corticalis, keine Vakuolen' fillde", und Fl emming sagt: „Ich habe vielfach grosse Rindenpartien der Drüsen ganz ohne Sekundarknötchen' getroffen." Bei den übrigen $90 \%$ der untersuchten Pferdelymphdrüsen waren Keimzentren in mehr oder minder grosser. Zahl za sehen: Diè Lymphdrüsen des Magens und Darmes verfügen relativ über die grösste Menge, allerdings nur relativ, da diese Drüsen eine wohlausgebildete Marksubstanz besitzen und dadurch die Keimzentren mit der Rinde oft auf einen schmalen Streifen zurückgedrängt werden, wenigstens was die Darmdrüsen angeht. - Die Deutlichkeit derkeimzentren ist auch eine recht verschiedene. (cf. Fig. 1). Die Mehrzahl ist undeutlich und verwaschen, wovon ungefähr $60 \%$ aller Drüsen und zwar in der Gesamtheit ihrer Keimzentren betroffen werden, sodass also nur bei etwa $30 \%$ die Keimzentren sich deutlich gegen die Umgebung abheben. Und auch hier sind vielfach die Umrisse nicht nach allen Seiten hin scharf gezeichnet, sondern die dem Zentrum des Sekundärknotens zugewandten Partien sind nicht so deutlieh begrenzt wie die dem Sinus zugekehrten. Die Ursache hierfür ist wohl in der variierenden Zellfiillung der Keimzentren zu suchen, indem sich die Lymphzellen ihrem Bestimmungsort den Lymphwegen - in erhöhtem Masse zuwenden, wozu vermutlich eine besondere Druckmechanik die Veranlassung bietet. In diesem Sinne erklärt auch Flemming die Wanderung der Zellen aus dem zentralen Teile durch die Peripherie nach aussen; er sagt: „Es muss in den Keimzentren eine Art von langsamer, zentrifugaler Druckmechanik bestehen, auf der es beruht, dass die jungen Tochterzellen nach der Peripherie zusammengedrängt und weiter durch die Lücken des Retikulums herausgetrieben werden."

Hierdurch lässt sich auch eine Erscheinung erklären, die darin besteht, dass im Innern mancher Keimzentren, wie ich gefunden habe, die Lymphzellen deutlich in zwei verschieden dichtgekörnten Zonen auftreten, nämlich in einer dunkleren (zellreicheren), halbmondförmigen und einer ovalen oder rundlichen, helleren Partie (cf. Fig. 1; kz). Fasst man diesen Wechsel im Auftreten 
der Keimzentren, ihren verschiedenen Füllungsgrad, vor allem aber den Umstand ins Auge, dass die weitaus grösste Zahl der Keimzentren beim Pferde nur ein verschwommenes Bild giebt, so ist der Gedanke nicht abzuweisen, dass diese Thatsachen Stützen für die Fle m ming'sche Hypothese bilden, ,dass nämlich die Keimzentren fluktuierende Dinge sind, welche temporăr auftreten, aus kleinen Anfangen anwachsen und sich, nach verschieden langem Bestehen, wiederum verkleinern und verlieren können." Es bleibe auch nicht unerwähnt, dass bei dem ungefähr 25jährigen Tiere nur $17 \%$ der Drüsen deutliche Keimzentren besassen, dagegen derselbe Prozentsatz von Lympknoten $(17 \%)$ ohne dieselben angetroffen wurde und in den übrigen die Keimzentren zum Teil kaum nachweisbar waren.

Die Gestalt der Keimzentren ist vorwiegend rundlich, doch findet man unter den Sinus öfters Formen, deren Breite ihre Höhe um das Doppelte übertreffen kann. (cf. Fig. 1).

\section{b. Marksubstanz.}

Es wurde bereits betont, dass durch das schwankende Verhältnis von Rinde und Mark erhebliche Strukturunterschiede bedingt sind. Diese Differenzen kommen hauptsalchlich durch das stark veränderliche Yerhalten der Marksubstanz zustande, indem diese bald an dieser bald an jener Stelle in die Rindensubstanz vordringt, auch wohl Partien derselben aus dem Zusammenhang mit dem anderen lymphoiden Gewebe loslöst und wie einen Kern umschliesst. Man gewinnt den Eindruck, als ob der Marksubstanz ein aktiv gestaltendes Prinzip innewohnt, freilich indirekter Natur, da in letzter Linie wohl die Lymphbahnen bestimmend auf ein Variieren einwirken dürften. $\mathrm{Zu}$ weilen erstreckt sich die Marksubstanz stellenweise bis direkt unter die Kapsel, was ich bei einem Fünftel aller Pferdelymphdrüsen der verschiedensten Gebiete beobachten konnte. (cf. Fig. $1, \mathrm{~m}, 0)$. In beschränltem Sinne haben hierauf schon einige Autoren hingewiesen, deren Angaben sich aber meist auf die Verhăltnisse beim Hunde beziehen; nur Sussdorf giebt an: „Vielfach erreicht an Stelle der austretenden Lymphgefässe die Marksubstanz selbst die Oberfläche; das sehen wir z. B. oft beim Pferde, Schafe und besonders beim Hunde." Diese Angabe stützt sich wahrscheinlich vor allem auf diejenigen Fälle, in 
welchen der Hilus fehlt. Das oben auf Grund meiner Untersuchungen mitgeteilte Resultat jedoch, dass bei $20 \%$ aller Drüsen die Marksubstanz bis direkt unter die Kapsel sich erstreckt, schliesst besonders jene Drüsen mit ein, wo die Marksubstanz, ausser im Gebiete des Hilus oder der austretenden Gefăsse, vor allem an peripheren Stellen vorhanden ist und hier die Sinus erreicht, sodass bisweilen ein Zusammenfliessen dieser mit den die Stränge umscheidenden Lymphbahnen sich ereignet. Zuweilen trifft man auch mitten im breiten lymphoiden Gewebe eine Andeutung der Marksubstanz in Gestalt weniger kurzer Stränge. Es handelt sich hierbei zweifellos um die peripheren Anfänge eines Markgebietes.

Bezüglich der Breitenausdehnung der Marksubstanz in höherem oder geringerem Grade sind verschiedene Litteraturangaben zu finden. Unter anderem äussert sich Frey über die Markmasse: „Dieselbe bietet bei der mikroskopischen Analyse manche Differenzen dar, verhält sich anders bei jungen Geschöpfen, wo sie oft allein in voller Ausbildung vorzukommen pflegt, als bei älteren und greisen Körpern, wo sie mehr oder weniger verkümmert getroffen wird. Ebenso zeigt sie nach den einzelnen Säugetierarten gewisse Verschiedenheiten. Endlich bietet die Marksubstanz in den inneren, namentlich den dem Verdauungskanale angehörenden Lymphknoten in der Regel eine höhere Ausbildung und Entwicklung dar als in den ausserlich gelegenen." In ähnlichem Sinne erläutert Rauber (19) seine Ansicht: „Die Marksubstanz ist in verschiedenen Drüsen ungleich mächtig entwickelt, am besten in den im Innern des Körpers gelegenen Drüsen, wie in den Mesenterial- und Lumbaldrüsen, während sie bei den oberflächlicher gelegenen (Achsel- und Leistengegend) mehr zurücktritt." Rauber bezieht sich nicht speziell auf die Lymphdrüsen der Säugetiere; aber es ist schon aus den Abbildungen $\mathrm{zu}$ entnehmen, dass er die Verhälnisse bei Tieren, wie das in allen human-medizinischen Lehrbüchern auf diesem Gebiete geschehen ist, zum grossen Teile zur Grundlage seiner Besprechungen gemacht hat.

Die Prüfung dieser Ausführungen an der Hand meiner Präparate ergab, dass man die $L y m p h d r u ̈ s e n ~ n a c h$ der Stärkedes A f fretensder Marksubstanzin Gruppen ordnen kann. Am ausgeprägtesten tritt dem Beobachter die 
Vergleichende Untersuchungen über den mikroskopischen Bau etc. 481

Marksubstanz an den Lymphdrüsen des Darmkanals entgegen, wo sie sich durch besondere Schönheit auszuzeichnen pflegt; weniger ausgebildet ist sie schon bei den Drüsen der Leber und Milz, sowie den Lenden-, Nieren- und inneren Darmbeindrüsen. Hierauf folgt zunächst die Gruppe der äusserlich gelegenen Lymphknoten, in welcher die Schamdrüsen noch am reichlichsten Markgewebe aufzuweisen haben, dann nimmt dieses immer mehr ab bei den Ellenbogen-, Achsel-, Kniekehlen-, Kniefalten-, Leisten- und Brustwanddrüsen, den Hals-, Bug-, Submaxillar-, Retropharyngeal- und ausseren Darmbeindrüsen. Die dritte Gruppe, in der also die Lymphstränge am wenigsten in die Erscheinung treten, setzt sich zusammen aus den Lg. bronchiales, mediastinales und gastricae. Vergleicht man diesés Ergebnis mit demjenigen, welches auf die Verteilung des Stützgerüstes Bezug hat, so sieht man, dass bis zu einem gewissen Grade allerdings das erhöhte Auftreten von Bindegewebszügen ein Zurückgehen der Marksubstanz im Gefolge hat. Doch beweist die dritte Drïsengruppe der letzten Vergleichsreihe, dass man eine diesbezügliche Regel durchaus nicht auf sämtliche Körpergebiete in Anwendung bringen kann. Vorzüglich überrascht das Verhalten der Lg. gastricae, welches erheblich von dem der übrigen Drüsen des Darmtraktus abweicht. Zur Prüfung dieser etwas befremdenden Thatsache wurden Serienschnitte durch eine ganze Magendrüse gelegt; durch diese Serie wurde ebenfalls die extreme Stellung der Lg. gastricae bewiesen; denn die Marksubstanz trat in ihrer geringen Ausdehnung gegenüber der übrigen breiten Drüsenmasse weit zurück.

Bei denjenigen Lymphdrüsen, welche über ein starkes Gerüst und gleichzeitig einen deutlich ausgeprägten Hilus verfügen, kommt es oft vor, dass durch das stark verzweigte Hilusstroma breitere Stränge aus der Rindensubstanz ausgeschnitten werden und so eine Marksubstanz vorgetäuscht wird. (cf. Figur 2, $\mathrm{r}^{\text {1.). }}$. Man ist nicht berechtigt, diese Bildung unter Hinweis auf die Definition der Marksubstanz, dass diese nämlich aus Strängen gebildet werde, als echte Marksubstanz anzusprechen, da in ihr vor allem hier und da Keimzentren auftreten und diese ein spezifischer Bestandteil der Rindensubstanz sind, da ferner neben diesen breiten Strängen in einigen Fällen eine echte Marksubstanz 
vorhanden ist, die sich durch geringe Breite der Stränge und Reichtum der Lymphbahnen auszeichnet.

Dass das Alter auf die Lymphdrüsenfunktion und -struktur grossen Einfluss ausübt, ist durch verschiedene Spezialarbeiten bewiesen worden (Billroth (20) u. a.). Da es sich hierbei meist um senile Erscheinungen handelt und die pathologische Seite in den Vordergrund rückt, so finde hier nur obige Ansicht Freys ihre Bestätigung, welche ausdrückt, dass im höheren Alter die Marksubstanz schwindet. Es ist leicht zu begreifen, dass die solidere Rindensubstanz dem Druck des wuchernden Bindegewebes höheren Widerstand zu bieten vermag als die locker gebaute Marksubstanz, und so ist bei den äusseren Körperlymphdrüsen namentlich eine Altersatrophie in dieser Richtung $\mathrm{zu}$ bemerken.

Eine vielumstrittene Frage ist von jeher die der Untersuchung über Entstehung und Bestandteile des Reti$\mathrm{kulums}$ oder Zellennetzes gewesen. In neuerer Zeit haben sich auf diesem schwierigsten Gebiete, welches das Lymphdrüsenstudium dem Forscher eröffnet, vor allem Hoyer (21) und Saxer (22) verdient gemacht, von welchen namentlich letzterer den Charakter der Retikulumzellen zu ergründen versucht hat.

In dieser Arbeit wird nur die eine Frage behandelt, ob die einzelnen Lymphdrüsen ein verschieden gebautes Retikulum zeigen. Hierzu habe ich die aufgeklebten Schnitte einiger ausgewăhlter Drüsen verschiedener Regionen einem stark mit Wasser verdünnten Pankreasglycerinextrakt (1:30) zum Zwecke langsamer Verdauung überlassen. (Hoyer verwendete einen solchen von $10 \%$ ). Die Verdauung war nach etwa 6 Stunden soweit vorgeschritten, dass die Lymphzellen fast vollkommen entfernt und das Stützgerüst sowie Retikulum allein zurückgeblieben waren. Obwohl sich die Pferdelymphdrüsen für die angegebene Darstellungsmethode des Retikulums nicht gerade zu eignen scheinen, lassen meine Schnitte doch erkennen, dass prinzipielle Unterschiede im Bau des Zellennetzes der einzelnen Lymphknoten offenbar nicht vorhanden sind.

Zum Schlusse möchte ich noch die Wege, welche die Lymphe in den Drüsen des Pferdes einschlägt, einer kurzen Besprechung unterwerfen. Die Lymphbahn zeigt in den einzelnen Drüsen erhebliche Schwankungen. Zunächst findet man, wie auch Sussdorf schreibt, dass die die Sekundärknoten umfassenden Lymph- 
Vergleichende Untersuchungen über den mikroskopischen Bau etc. 483

sinus bald schmäler bald breiter sind, was sich nach dem jeweiligen Füllungszustand der zuführenden Lymphgefässe hauptsächlich richten dürfte. Dementsprechend findet man bei geringer Lymphmenge die Sinus kollabiert, und umgekebrt bilden sie bei stärkerer Füllung helle Umsäumungen um die lymphoiden Massen.

Wie bekannt, setzen sich die Sinus in die Lymphgänge der Marksubstanz fort und treten als solche hier mehr oder weniger zahlreich je nach der Ausbreitung der Marksubstanz auf, sodass die Lymphwege in den Drüsen des Darmkanals naturgemăss ihre höchste Entwicklung erfahren. Es muss hierbei noch eines ganz besonders wichtigen Umstandes Erwähnung gethan werden, auf den bis jetzt als einziger Sussdorf aufmerksam gemacht hat, und welcher deshalb so gut wie unbeachtet geblieben ist, weil die Lymphdrüsen des Pterdes nur selten Gegenstand eingehender Untersuchungen gewesen sind. Sussdorf schreibt: „Andere Lymphdrüsen des Pferdes lassen dagegen einen eigentümlich kavernösen Bau erkennen; in Durchschnitten solcher finden sich zahlreiche, durchschnittlich $5 \overline{5}-78$, grosse, auch konfluierende, rundliche Lücken, welche von Endothelien umgrenzt sind, aber einer eigentlichen Wand entbehren. Zwischen ihnen lagert fast allein lymphoides Gewebe, das somit in Form von feineren und gröberen netzförmig verbundenen Scheiden auftritt, welche die Lymphbahnen - denn als solche erweisen sich in Injektionspräparaten die angedeuteten Kavernen - umhüllen." Man findet häufig in der Rindensubstanz diese Lücken, wo sie die verschiedensten Formen zeigen; teils sind sie rundlich oder mehr eckig, teils spaltenförmig. Ich habe nun feststellen können, dass diese Lücken vielfach mit schmalen kavernösen, teilweise retikulumhaltigen Lymphgängen in Verbindung stehen, die sich nach der Marksubstanz hinziehen und hier mit den Lymphgängen des Marks ein mannigfach verzweigtes Netz bilden. Es ergiebt sich hieraus, dass den Pferdelymphdrüsen ausser dem bekannten System der Sinus und Lymphgänge ein zweites System von Kavernen und kavernösen Gängen zukommt, welches sich mitten durch das lymphoide Gewebe zieht, ohne von Gerüstzügen begleitet zu sein. (cf. Fig. 3, c.)

Dieses frei die Drüse durchziehende Lymphspaltensystem konnte ich in ungefähr $20 \%$ aller Drüsen nachweisen, woraus 
hervorgeht, dass es sich nicht $u m$ eine selten $z u$ beobachtende Erscheinung, sondern um eine besonders für die Pferdedrüsen charakteristische Einrichtung handelt, die ihre beste Ausbildung in den gerüstarmen Drüsen des Darmes, der Leber und Milz findet. Dieser letztere Umstand berechtigt zu der Annahme, dass der geringeren Ausbildung des Septensystems wegen ein Teil der Lymphbahnen. gezwungen wird, in das Parenchym einzudringen, ohne dem Verlaufe bahnbrechender Septen folgen zu können.

Rind.

Die Kenntnisse über den histologischen Bau der Lymphdrüsen des Rindes sind von jeher durch eingehendes Studium mehr gefördert worden als diejenigen der Lymphdrüsen anderer Tiere. Doch haben sich die diesbezüglichen Forschungen, wie in der Einleitung schon bemerkt wurde, nur auf eine beschränkte Zahl von Lymphgebieten erstreckt, und die bahnbrechende Arbeit von $\mathrm{H}$ is ist bis heute, streng genommen, grundlegend geblieben für die Untersuchungen seiner Nachfolger auf diesem Gebiete. Somit bestand meine Aufgabe bei der Untersuchung des Baues der Rinderlymphdrüsen in der Hauptsache darin, die vorhandenen Angaben zu prüfen, vor allem aber festzustellen, ob und in welchem Massedie namentlich für die Inguinal-, Axillar- und Mesenterialdrüsen überlieferten Befunde sich auf die Lymphdrüsen aller anderen Gebiete übertragen lassen. Zu dem Zwecke habe ich ausser einer grösseren Zahl von mir angefertigter Schnitte die Lymphknoten aller Gebiete von drei Rindern mikroskopisch untersucht, mit Ausnahme der Leisten- und Milzdrüsen, die bei den Versuchstieren nicht gefunden werden konnten, und von denen erstere bei 7 , letztere bei 15 weiteren Rindern vermisst wurden. Die mikroskopischen Untersuchungen haben nun zu folgenden Ergebnissen geführt.

\section{Stiitzgerüst.}

Die Kapsel und die Septen zeigen bei der Mehrzahl aller Rinderdriisen eine hohe Ausbildung. Erstere ist meist von ganz beträchtlicher Stärke, und von ihr gehen häufig entsprechend starke Scheidewände (Trabekeln) ab, welche einen hoch entwickelten alveolären Bau der Lymphknoten bedingen. Vor allem 
zeichnet sich aber in der überwiegenden Zahl aller Rinderdrüsen die Marksubstanz durch ein wohl ausgeprägtes Septennetz aus, das mit dem Stroma des Hilus, bezw. der bei dessen Fehlen stark verdickten Kapsel in engstem Zusammenhange steht. v. Recklinghausen ist sogar der Ansicht, "dass in den Lymphdrüsen des Ochsen.....jeder Abschnitt der Lymphbahn in seiner ganzen Längevon einem Trabekel durchzogen ist." Es trifft das bei den von mir untersuchten Drüsen vielfach $\mathrm{zu}$, aber durchaus nicht immer, was besonders betont sei. (cf. Fig. 5 u. 6, sn.)

Sehr reichlich sind, wie auch ich gefunden habe, Züge glatter Muskulatur in Kapsel und Septen der Drüsen des Rindes zugegen, worauf zuerst Malpighi, später Heyfelder (23), besonders aber $\mathrm{H}$ is hingewiesen haben. Alle späteren Autoren, z. B. Schwarz (24), schliessen sich dieser Ansicht an mit Ausnahme von Teichmann, welcher schreibt: „Die Angabe von $\mathrm{H}$ is, dass in den Drüsen ein grosser Reichtum an glatten Muskeln vorhanden sei, kann ich nicht bestätigen."

Wie in allen Lymphdrüsen überhaupt, so sind auch in denen des Rindes Kapseln und Septen in Bezug auf ihre Stäre erbeblichen Variationen unterworfen, worin sich jedoch eine gewisse Regelmäsigkeitgeltend maclit. An der Hand meiner Präparate kann ich die Ansicht früherer Autoren bestătigen, dass die inneren Drüsen im allgemeinen ein geringer entwickeltes Stützgerüst besitzen als die äusserlichen. Doch möchte ich noch hinzufügen, dass, obwohl gerade beim Rinde viele Ausnahmen hierin vorkommen, gewöhnlich Lenden-, Nieren-, Leberund Darmdrüsen an der Spitzedergerüstärmeren Ly mphknoten stehen, und dass zu den ger üstreicheren Achsel-, Bug- undKniekehlendrüsen die übrigen den Uebergang bilden. (cf. Fig. 4 u. 6, K, s.)

Obwohl ich betreffs des Auftretens eines Hilus gleichfalls bedeutende Schwankungen angetroffen habe, war es mir nicht möglich, auch hierbei irgend eine bei gewissen Drüsen sicher wiederkehrende Beschaffenheit des Hilus festzustellen. Es dürften sich die vorkommenden Unterschiede auf alle Regionen ungefähr gleichmässig verteilen. Diese Auffassung möchte ich auch den Ausführungen von $\mathrm{His}$ gegenüber 
aufrecht erhalten, wenn er sagt: „Bei den Inguinal- und Axillardrüsen des Rindes ist . . . . die Marksubstanz stark entwickelt, während das Hilusstroma eine nur untergeordnete Rolle spielt." Die genannten Drüsen zeigten nur bei einem der drei von mir herangezogenen Rinder diese eben von $\mathrm{H}$ is geschilderte Anordnung, während bei den zwei anderen Tieren gegenteilige Verhältnisse $\mathrm{zu}$ bemerken waren.

Einen gut entwickelten Hilus (cf. Fig. 4, h). fand ich bei etwa $70 \%$ aller Rinderdrüsen, bei $30 \%$ war er nur durch eine entsprechende Kapselverdickung angedeutet. (cf. Figur 6, h.) Eine Besonderheit in der Ausbildung des Hilus besteht nach meinen Untersuchungen bei manchen Rinderdrüsen darin, dass das Hilusstroma sich in Gestalt einer solchen Kapselverdickung mehr bogenförmig in die Markmasse einbuchtet (cf. Figur 5, h.). Jedoch giebt es auch hier keine Gebiete, bei deren Drüsen diese Erscheinung vorzugsweise aufträte.

\section{Parenchym.}

Der Ausspruch v. Recklinghausens: „Auch der feinere Bau ist beim Rind in seinen Einzelheiten am präzisesten ausgesprochen, am leichtesten zu erkennen" trifft bei meinen sämtlichen Präparaten vorzüglich für das Parenchym zu; denn ich habe bei allen Drüsendieschematische Forderung der Verteilung von Rinde und Mark erfüllt gesehen, ja in manchen Lymphknoten traf ich sogar eine deutliche Schichtung beider Substanzen, indem, unter Zugrundelegung der Stellung des Hilus in der Drüse, die Rindensubstanz die obere (dorsale) und die Marksubstanz die untere (ventrale) Lage des Parenchyms bildeten (cf. Fig. 6, m, r.). Man findet somit bei den Rinderdrüsen mit geringen Ausnahmen die beiden Substanzen in deutlicher Entwicklung neben einander vor. "Doch sind“, wie $\mathrm{H}$ is treffend schildert, „Rinden- und Marksubstanz nirgends scharf von einander geschieden; sie greifen vielfach in einander, und während einzelne Rindensegmente oft gegen den Hilus hin sich verlängern, kann es auch kommen, dass Fortsätze der Marksubstanz sich weit in die Rinde hinein vorschieben."

Nur selten, nämlich in ung ef äh $10 \%$ der D ü sen, sah ich schmale Markstreifen die Rinde an einer Stelle durchbrechen und bis an die Drüsenoberfläche vor- 
dringen, wo dann die Marksubstanz, stets in nur geringer Ausdehnung, zu Tage trat. Was nun die Beziehung der beiden Substanzen betreffs ihrer Breitenausdehnung angeht, so kann ich mich auf Grund meiner Schnitte der in den allgemeinen Besprechungen der Lehrbücher der Histologie wiedergegebenen Auffassung nicht anschliessen, dass die inneren Drüsen eine höher entwickelte bezw. breitere Marksubstanz besitzen sollen als die äusseren. Nach meinen Schnitten scheint in diesem Sinne eine Unterscheidung der Drüsen des Rindes nicht in dem Umfange durchführbar zu sein; denn ich habe bei ungefähr ebensoviel äusseren Lymphknoten eine ausgeprägte und ausgedehnte Marksubstanz beobachtet als bei den im Innern des Körpers befindlichen, und umgekehrt. Wie ich in der Besprechung der Pferdelymphdrüsen schon angedeutet habe, sind Rinde und Mark in ihrer Breite von einander abhängig; das trifft natürlich für die Drüsen des Rindes ebenfalls zu. Und so konnte ich auch bezüglich der Rindensubstanz regelmässig bei den einzelnen Lymphknoten verschiedener Regionen sich wiederholende Abweichungen nicht konstatieren. Ich möchte daher meine Ansicht über die Verteilung der beiden Parenchymsubstanzen in den einzelnen Rinderlymphdrüsen folgendermassen zusammenfassen: $\mathrm{R}$ in d e nund Marksubstanz sind, trotzdem sie in ihrer Anordnung meist einem regelmäsigen Bau entsprechen, beträchtlichen Schwankungen unterworfen, welche jedoch bei den Lymphknoten aller Gebiete ungefahr gleich stark zur Beobachtung g e l a ng en.

Wie die Rindensubstanz der meisten Lymphdrüsen von Tieren anderer Gattungen ist dieselbe auch beim Rind durch das Auftreten von $\mathrm{K}$ e i $\mathrm{m}$ e $\mathrm{n} \mathrm{tr}$ e n ausgezeichnet, die aber bei diesem Tiere grössere Formverschiedenheiten darbieten als bei jenen, insofern als sie häufig in zur Drüse zentripetaler Richtung eine besondere Ausdehnung erlangen und so mehr langgestreckte Gestalt annehmen oder auch auf Durchschnitten mit dem Bau einer Pyramide sich vergleichen lassen, deren Basis peripher, deren Spitze zentral zu liegen kommt. I $\mathrm{ch}$ habe die Keimzentren, wenn auch bisweilen undeutlich und verschwommen, in allen Schnitten durch Rinderdrüsen gesehen und habe im Mittel 2,38 Keimzentren im Gesichts- 
feld gezahlt (Zeiss, Okular 2, Objektiv A), die durchschnittlich $0,276 \mathrm{~mm}$ breit waren. Dem Auftreten der Rindensubstanz entsprechend wird offenbar keine Lymphdrüsensorte mit einer besonders hohen oder niederen Zahl von Keimzentren bedacht.

In Bezug auf die Marksubstanz der Rinderdrüsen sei erwahnt, dass man an ihr besonders schön die Entstehung der Marksträngeausden Sekundärknoten beobachten und ihren Verlauf auf dem Wege nach dem Hilus verfolgen kann. (cf. Fig. 5, ms.) Diese bekannte Thatsache kleidet v. Recklinghausen in folgende Worte: „In den Lymphdrüsen des Ochsen sind Lymphbahn und Follikulargewebe schon deswegen genau unterschieden ......., weil das Follikulargewebe auch durch die ganze Marksubstanz hindurch kontinuierlich zusammenhängende, nicht unterbrochene Balken bildet, welche an Breite die Lymphbahnen meistens ïbertreffen." Diese Darstellung könnte geeignet sein, den Glauben zu erwecken, als ob alle oder wenigstens die Mehrzahl der Markstrănge als zusammenhängende Parenchymstreifen bei mikroskopischer Betrachtung im Schnitt zu erkennen seien. In Wirklichkeit fand ich dieses Verhalten der Markstränge in den Rinderdrüsen nur häufiger und vollendeter als in den Lymphknoten anderer Individuen, sodass immer noch die Mehrzahl der Markstränge beim Rinde mit denen anderer Tiere darin übereinstimmt, dass die Strange sich infolge ihres gewundenen Verlaufes im Präparate zerschnitten und mithin scheinbar unterbrochen darstellen.

Einer von mir in verschiedenen Rinderdrüsen beobachteten Eigentümlichkeit der Marksubstanz werde hier noch kurze Besprechung zu teil. Eingangs erwähnte ich, dass beim Rinde manche Lymphknoten eine deutliche Schichtung erkennen lassen. In diesen Drüsen sah ich, namentlich wenn der Hilus nur angedeutet war, dass die Markstränge nicht wie in anderen Drüsen dem Hilus oder dessen Andeutung sich zuwandten, sondern ein M a r k g e f l e c h t bildeten, d es se n Stränge mehr oder weniger $z$ ur Kapsel parallel verliefen, woran sich auch das Septennetz beteiligte, sodass die Längsrichtung der Maschen gleichfalls in zur Kapsel ziemlich parallele Ebenen zu liegen kam. (cf. Fig. 6, m. u. sn.)

Zur Darstellung des Retikulums wurden die Rinderdrüsenschnitte genau wie diejenigen vom Pferd behandelt, doch 
Vergleichende Untersuchungen über den mikroskopischen Bau etc. 489

gelang es bei den Lymphknoten des Rindes, unverletzte Retikulumpräparate des ganzen Schnittes zu erhalten. Aber auch für das Rind hat sich ergeben, dass die einzelnen Lymphknoten in der Struktur ihrer Zellennetze im wesentlichen übereinstimmen. Es sei jedoch hervorgehoben, dass die Retikulummaschen der Rindensubstanz im allgemeinen etwas weiter sind als in der Marksubstanz. (cf. Fig. 9.)

Die Lymphsinus schliesslich zeichnen sich häufig durch besondere Breite aus, was zum Teil mit der bedeutenden Grösse mancher Rinderdrüsen in Einklang zu bringen sein dürfte. Vor allem fand ich aber auch die Lymphgänge der Marksubstanz oft sehr weit und ausgedehnt, speziell am Uebergang der Rinden-in die Marksubstanz. (cf. Fig. 5, l.) Diese Lymphgänge verlieren nach dem Hilus zu an Weite und werden dann gewöhnlich von den Marksträngen an Breite übertroffen. - Ausserdem verlaufen mitten im Parenchym der Rinde in allen Drüsen kleine Lymphgefässe; endlich sei noch bemerkt, dass ich in einer oberflächlichen Inguinaldrüse verschiedene, kleinere Kavernen angetroffen habe.

\section{Schwoin.}

Zur Beurteilung des histologischen Baues der Schweinelymphknoten wurden neben einer grösseren Zahl von Schnitten, welche aus Drüsen verschiedener Schweine angefertigt worden waren, die sămtlichen Drüsen von drei Tieren herangezogen mit Ausnahme der Leisten- und äusseren Darmbeindrüsen, welche vermutlich wegen ihrer sehr unbedeutenden Entwicklung in dem starken Fettpolster nicht gefunden werden konnten. Vielleicht waren sie auch der fettigen Degeneration anheimgefallen, was bei vielen Schweinedrüsen der Fall ist. Die Drüsen des Dünndarms scheinen überhaupt zu fehlen, wenigstens waren sie bei keinem der zehn daraufhin untersuchten Tiere vorhanden.

Die Lymphdrüsen des Schweines haben das Interesse von nur sehr wenigen Forschern zu erwecken vermocht, wofür die geringe Zahl der Litteraturangaben am besten spricht. Und so kann es nicht Wunder nehmen, dass die eigenartige Stellung der Lymphdrüsen des Schweines in Bezug auf ihren histologischen Bau nirgends zur Genüge bervorgehoben worden ist. Die Untersuchung der von mir angefertigten Schnitte hat betreffs des histologischen Baues folgendes ergeben. 


\section{Stützgerüst.}

Kapsel und Septen bestehen aus fibrillärem, glatte Muskelfasern haltigen Bindegewebe, in dem hier und da Lücken und Spalten auftreten, die mit Lymphzellen angefüllt sind. (Klein [25]). Sussdorf hebt noch hervor, dass Hülle und Gerüst „besonders beim Schweine auch durch ein zartes, netzadriges Gewebe, in welchem ausserordentlich zahlreiche, undeutlich abgegrenzte Zellen mit grossen rundlichen Kernen sich finden", hergestellt würden. Der Anschauung, dass dieses fragliche Gewebe zum Gerüst gehöre, kann ich nicht beipflichten, und ich werde später versuchen, Beweise für meine gegenteilige Ansicht $\mathrm{zu}$ erbringen.

Die Kapsel ist im allgemeinen bei allen Lymphdrüsen des Schweines schwach entwickelt, und das Septensystem zeigt insofern eine Abweichung von der sonst gültigen Regel, als es Verbindungen mit der Kapsel nur in ganz geringem Umfange eingeht. Dafür springt eine andere Erscheinung um so mehr in die Augen; die Schnitte durch die Schweinelymphdrüsen sind von mehr oder weniger rundlicher oder ovaler Form. Im Innern dieser Schnitte fand ich nun häufig ein stärkeres, gefässhaltiges Septum, um welches die wenig unter einander sich verbindenden, hirschgeweihähnlichen Septen gelagert sind und zwischen sich und Kapsel, bezw. Sinus, gewöhnlich einen breiteren, ringförmigen Parenchymstreifen lassen, welcher, wie schon erwähnt, nur selten von Scheidewänden durchzogen wird. (cf. Fig. 7, $\mathrm{r}^{1}, \mathrm{r}^{2}$.) In neuester Zeit schreiben Ell e n b e r g er und Gü $n$th er (26) über das Gerüst: "Mächtiger ist es in der Marksubstanz beim Schafe und Schweine." Genannte Autoren haben offenbar diese zentralen, stärkeren Septen bei ihren Besprechungen im Auge gehabt. Ich bin aber nicht der Ansicht, dass das Gewebe, in welchem die Gerüstzüge sich befinden, zur Marksubstanz gehört, sondern es ist meines Erachtens zur Rindensubstanz zu rechnen; denn die Marksubstanz ist beim Schweine, wie später gezeigt werden soll, sebr kümmerlich entwickelt und führt nur wenig, ganz schmale Septen. Die Stärke der mehr zentral befindlichen Septen schwankt wie die Dicke der Kapsel, und ich habe versucht, hiernach die Lymphknoten des Schweines zu ordnen. Eine gewöhnlich schwache Kapsel und geringe Septenentwicklung zeigen die Drüsen des Magens, Darmes, Gekröses, der Milz und Leber; stärker ist das Gerüst schon bei den Lenden-, Nieren- und inneren Darmbein- 
Vergleichende Untersuchungen über den mikroskopischen Bau etc. $49 \mathrm{I}$

drüsen sowie denjenigen der Bronchien, des Mediastinums und der Brustwand. Am reichlichsten tritt die Stützsubstanz bei den äusserlich gelegenen Drüsen auf und $z$ war verstärkt sie sich ungefähr entsprechend der Reihenfolge, in der die Drüsen genannt. werden, nämlich Retropharyngeal-, Hals-, Submaxillar-, Bug-, Kniekehlen-, Kniefalten-, Scham- und Achseldrüsen.

Ein eigentliches Hilusstroma fand ich sehr selten. Man sieht meistens nur an einer oder mehreren Stellen die Kapsel verstärkt und gefässhaltig, aber nicht eingesenkt. (cf. Fig. 7, h.) Der Hilus ist also in der Regel nur ande utungs eis e vorhanden. - Eine interessante Bildung beschreibt $\mathrm{Ch}$ ievit z in seinem Spezialartikel über die Mesenterialdrüsen des Schweines wie folgt: „Bei den uns jetzt beschäftigenden Drüsen des Schweines dagegen senken sich die Vasa lymphat. inferentia durch einen bindegewebserfüllten Spalt in das Innere der Parenchymschale ein, während die abführenden Stämme und die grösseren Blutgefässstämme an der Aussenseite gelagert sind."

\section{Parenchym.}

Die lymphoide Masse der Lymphknoten zeigt eine zunächst schwer zu verstehende Anordnung. v. Recklinghausen sagt: „Die Lymphdrüsen des Schweines zeigen in einer Beziehung ganz entgegengesetzte Eigenschaften; in ihnen ist die follikuläre Sub$\operatorname{stan} z$ gegenüber der Lymphbahn vorwiegend; auch durch die ganze Marksubstanz hindurch erscheinen an den Follikularsträngen noch knotige Anschwellungen, also wirkliche Follikel, auf der Schnittfläche sieht man schon mit blossen Augen diese Körnchen vorspringen, die Lymphbahn ist infolgedessen so eng, dass die Injektion hier mit grossen Schwierigkeiten verknüpft ist. Nach Franz Schmidt finden sich auch an anderen Orten im Körper. des Schweines, im Rachen z. B., die follikulären Apparate besonders stark entwickelt, es bedarf noch weiterer Untersuchung, um festzustellen, ob in Folge der Mästung dieser Tiere, wie Schmidt vermutet, oder ob hierin eine Eigentümlichkeit dieser Tiergattung $\mathrm{zu}$ suchen ist."

Chievitz schreibt über die adenoide Substanz der Mesenterialdrüsen, "dass sie in den zentralen Teilen der Drüse in rundlichen Abteilungen vielfach ausgeschnitten wird, sich gegen die Peripherie hin in eine ganz zusammenhängende Lage fortsetzt, . 
die nur stellenweise von Trabekeln durchzogen wird." Nach meinen Untersuchungen ist, wie oben schon gesagt wurde, in den allermeisten Drüsen des Schweines diese Anordnung die nämliche, und ich will versuchen, das Resultat der Durchmusterung meiner Präparate im folgenden wiederzugeben.

Bei mikroskopischer Betrachtung irgend eines Drüsenschnittes, gleichgültig aus welcher Körperregion die Drüse genommen ist, fallt sofort ein deutlicher Unterschied in der Anordnung des Parenchyms auf. Es giebt eine zellreiche lymphoide Substanz, in der die Keimzentren auftreten, welche also der Rindensubstanz der Lymphknoten anderer Tiere entspricht, und ausserdem hellere, mehr band- oder streifenförmige Partien, die weniger Lymphzellen beherbergen. (cf. Fig. 7, $\mathrm{r}^{2}$.) Letztere allein kann Sussdorf mit dem "zarten, netzadrigen Gewebe", in dem viele Zellen sich finden, gemeint haben. Diese beiden leicht von einander zu unterscheidenden Gewebe zeigen nun eine allen Lymphknoten des Schweines zukommende, eigenartige Verteilung. Die keimzentrenhaltige Substanz liegt im allgemeinen mehr im Innern der Lymphdrüse, in geringerem Umfange tritt sie bis unter die Kapsel (cf. Fig. 7, K.); sie bildet mithin den Kern des Lymphknotens, den die zellärmeren Partien ringförmig umgeben (cf. Fig. 7, r $^{2}$ ) und vielfach in Form breiter Streifendurchsetzen, wohl auch Inseln aus der dichten lymphoiden Masse ausschneidend. In manchen Drüsen überwiegt diese hellere Substanz ganz erheblich. Beide Gewebsarten sind aber weder durch Septen, Blutgefässe noch Lymphbahnen von einander geschieden, sondern gehen ausnahmslos ohne Grenze allmăhlich in einander über. Vielfach treten auch streifenartige oder mehr rundliche Zellanhaufungen, die jedoch mit Keimzentren nichts gemeinsam haben, in der helleren Substanz auf, sodass hierdurch schon die Vermutung nahe gelegt wird, dass beide Gewebsarten verwandt sind. Zur Beantwortung dieser Frage lube ich aufgeklebte Schnitte nach der oben angegebenen Methode, für welche sich die Lymphdrüsen des Schweines besonders gut zu eignen scheinen, der künstlichen Verdauung unterworfen, teils mit Fuchsin-Resorzin und hierauf mit Säurefuchsin-Pikrinsäure, teils mit Phosphormolybdänsäure-Hämatoxilin (S c h morl [27]) gefärbt und Dauerpräparate angefertigt. An der $\mathrm{H}$ and dieser Retikulum- 
Vergleichende Untersuchungen über den mikroskopischen Bau etc. 493

präparate ist es mir gelungen, den Beweis $z$ u. liefern, dass die hellere, streifenförmige substanz retikuläres Bindegewebe zur Grundlage: hat, und dass dieses in das Retikulum der eigentlichen Rindensubstanz ohne irgend welche Grenze. übergeht. (cf. Fig. 10.) Beide Zellennetze unterscheiden sich nur dadurch, dass dasjenige der helleren Partien engmaschiger und in seinen einzelnen Elementen stärker gebaut ist als das. Retikulum des dichtgekörnten Teils der Rindensubstanz. (cf. Fig. 10 und 11.) Ausserdem finden sich in ersterem reichlicher kleine Gefässe. Dieser Bau ist, wie die Struktur der Zellennetze überhaupt, in allen untersuchten Drüsen der nämliche, sodass histologische Verschiedenheiten in der Retikulumstruktur der einzelnen Lymphknoten offenbar nicht bestehen. $\mathrm{Da}$ in dem dichteren Retikulum, wie in dem zarter gebauten, Lymphzellen auftreten, so gehört die hellere Drüsensubstanz gleichfalls zu dem adenoiden Gewebe, also nicht zum Gerüst. Somit besteht die Rindensubstanz aus einem zellenreichen, keimzentrenhaltigen und einem zellärmeren, lymphoiden Gewebe, welches der Keimzentren entbehrt. Diese Rindensubstanz bildet in den Lymphknoten des Schweines denweit überwiegenden. Ha u tbestandteil des ganzen Parenchyms. (cf. Fig. 7.)

Die Keimzentren selbst erfahren beim Schwein eine gleichmässig hohe Entwicklung, sowohl was die Schärfe betrifft, mit der sie sich gegen das übrige lymphoide Gewebe abheben, als auch in Bezug auf ihre hohe Zahl. Ich habe bei schwacher Vergrösserung im Mittel 6,26 Keimzentren im Gesichtsfeld gefunden. Sie liegen in den zellreichen Partien der Rindensubstanz und hier meist dicht unter den Lymphwegen, welche sich um die Septen erstrecken (cf. Figur 7); seltener sáh ich sie unter den Sinus und in wenigen Fallen mitten im Parenchym. Ihren Durchmesser habe ich auf $0,242 \mathrm{~mm}$ - berechnet. Dagegen giebt. Sussdorf von den "Follikeln" an: "beim Schweine stellen sie deutlich runde oder ovoide Bläschen dar, die durchschnittlich $0,35 \mathrm{~mm}$ messen." Die Form der Keimzentren ist allerdings fast ausschliesslich rund, und ihre Begrenzung ähnelt meist der eines. scharf gezogenen Kreises, so dicht und deutlich treten die peripheren Retikulumfasern um sie auf (cf. Fig. 7, k.). Seltener traf ich verschwommene Keimzentren, und nur in einer einzigen 
Drüse war es mir unmöglich, sie nachzuweisen. Ihr Füllungsgrad schwankt.

Der Reichtum an Keimzentren und die starke Ausdehnung der Rindensubstanz sind Eigenschaften fast aller Lymphdrüsen des Schweines. Die hohe Entwicklung der lymphoiden Bestandteile dürfte also kaum als Folge der Mästung anzusehen sein, sondern sie ist einfach ein Charakteristikum für die Lymphknoten des Schweines.

Eines Umstandes sei noch kurz gedacht. In einer Leberund einer Magendrüse bemerkte ich im zellenreichen Parenchym einige grosse Kavernen. . Bei Betrachtung mittels starker Vergrösserung liess sich deutlich erkennen, wie aus dem umliegenden lymphoiden Gewebe, auch aus den vorspringenden Keimzentren, Lymphkörperchen in die Kavernen übertraten.

Nach allgemein gültiger Regel steht im Gegensatz zur Rindensubstanz ein Markgewebe. Prüft man die Drüsenschnitte des Schweines auf dessen Vorhandensein, so ergiebt sich, dass die Marksubstanz eine überraschend geringe Ausbildung erkennen lässt. Ich konnte sie überhaupt nur bei $30 \%$ aller Drüsen nachweisen, und auch hier war sie in der Mehrzahl der Fälle nur andeutungsweise zu bemerken. Jedenfalls tritt sie im Verhältnis zur Rindensubstanz vollkommen in den Hintergrund. Sie liegt fast nur in den periphersten Partien der helleren Substanz, sodass man sie meist, allerdings in geringer Ausbreitung, dicht unter der Kapsel und zwar gewöhnlich in der Nähe der Hilusandeutung findet (cf. Fig. 7, m.). In einigen Fällen fand ich sie etwas ausgedehnter; sie zog sich unter den Sinus als ringförmige, äussere Schicht des breiten, zellarmen Parenchyms hin. Die Marksubstanz wird nämlich beinahe ausschliesslich ron Strängen dieser helleren Substanz gebildet. Die Stränge sind kurz und schmal und geben durch ihre Anastomosen Veranlassung zur Entstehung eines Netzes mit langgezogenen Maschen, welche von gleichfalls schmalen Lymphbahnen und einigen sehr gering entwickelten Gerüstzügen ausgefüllt werden. Hin und wieder sind die Markstränge auch zellreicher und ähneln dann in ihrem Bau dem dichtgekörnten cytogenen Gewebe. Ich kann mich aber der Ansicht v. Recklingha usens nicht anschliessen, dass man in der Marksubstanz „Follikel“, also Keimzentren, fände; das zellreiche Gewebe schiebt 
Vergleichende Untersuchungen über den mikroskopischen Bau etc. 495

sich nur ab und $z u$ in das zellärmere ohne scharfe Grenze ein. Letztere Gewebsart erwähnt v. Recklinghausen gar nicht.

Die Lymphbahnen zeigen gemäss dem vom allgemeinen Drüsenschema abweichenden Bau des Parenchyms auch eine charakteristische Entwicklung. Die Sinus fand ich ohne grundsätzliche Unterschiede bald breiter, bald schmäler. Aus dem oben Gesagten aber, nur selten gehen Septen von der Kapsel ab, lässt sich schliessen, dass die Sinus ohne bedeutende Unterbrechungen sich rund um das Parenchym unter der Kapsel erstrecken müssen und zwar bis zum Hilus. An dieser Stelle sei die von Frey in einer Anmerkung seines Handbuches niedergelegte Ansicht über diesen Punkt wiedergegeben. F rey schreibt, vermutlich unter Anlehnung an die Verhälnisse bei den Rinderdrüsen: „Nicht ohne Interesse ist die Möglichkeit eines rein oberflächlichen Lymphstroms durch den Knoten. Aus der Auflösung des Vas efferens leiten einzelne Bahnen alsbald nach Umhüllungsräumen der Follikel. Indem diese letzteren Hohlgänge, wie wir wissen, miteinander kommunizieren und in sie die Vasa afferentia sich einsenken, kann einströmende Flüssigkeit nur durch jene Umhüllungsräume unter Vermeidung der Lymphgänge des Marks zum Abfluss gelangen. Schon vor längeren Jahren hat Donders diese physiologisch nicht unwichtige Beobachtung gemacht, welche ich bei späterer Untersuchung bestätigen konnte." Dieser Gedanke ist bei Frey und Donders (28) durch logische Ueberlegung entstanden, aber wahrscheinlich haben beide keine Gelegenheit gehabt, an Präparaten aus Rinderdrüsen die Richtigkeit ihrer Anschauung zu erkennen. Mir ist das an Schnitten von Schweinedrüsen gelungen. An einigen ron ihnen lässt sich der zirkuläre Verlauf der Sinus ohne Unterbrechung verfolgen und die Kommunikation mit den zum Hilus fliessenden Lymphbahnen dicht unter der Kapsel einwandsfrei nachweisen (cf. Fig. 7, si). Vermutlich ist der oberflächliche Lymphstrom in den Drüsen des Schweines besonders ausgeprägt, worauf auch die peripher gelegene, zum Teil lang sich hinziehende Marksubstanz hindeutet.

An den die Septen umfliessenden Lymphsinus konnte ich keine Besonderheiten entdecken. Dagegen fiel mir die mit der geringen Entwicklung der Marksubstanz einhergehende Armut an Lymphbahnen überhaupt auf. Chievitz sagt hierüber bezüg- 
lich der Mesenterialdrüsen: "Wăhrend in anderen Drüsen gegen die Vasa efferentia zu die Lymphbahnen immer zahlreicher werden, das Parenchym daher in ein Geflecht von schlankeren Parenchymsträngen zerfallt, liegt in den Schweinsdrüsen unter der Oberflache ein breit zusammenhängendes Lager von Drüsensubstanz, welches nur hier und da von Lymphbahnen durchzogen wird."

Vor allem sei betont, dass es mir unmöglich gewesen ist, irgend einen Zusammenhang der Lymphbahnen der Marksubstanz mit denjenigen zu erkennen, welche die stårkeren Septen des Parenchyms umgeben. Ich sah vielmehr, dass die abführenden Lymphbahnen ihre Entstehung in dem zellarmeren Teile des Parenchyms nehmen. Die Retikulummaschen werden an manchen Stellen weiter, fliessen zu kleinen Lücken zusammen, und aus der Vereinigung mehrerer solcher Lücken entspringt eine feine Lymphbahn, die fast regelmässig im Zentrum des zellärmeren Parenchyms verlaufend ihren Weg nach den peripheren Sinus zu einschlägt (cf. Fig. 7, 1). Bisweilen finden sich mehrere solcher zarten Gefasse nebeneinander und bilden mit konvergierend zu ihnen laufenden Lymphwegen anderer hellerer Parenchymstreifen grössere, zum Hilus sich wendende Gefässe. - Es besteht aber trotz allem die Thatsache, dass die Schweinedrü sen an Lymphwegen sehr arm sind. Demgegenüber ist die Zellvermehrung, wie infolge des Reichtums an Keimzentren angenommen werden muss, eine hohe und die Frage somit naheliegend: auf welche Weise gelangen die Lymphzellen aus dem zellreichen Parenchym in die Lymphbahnen? Dort wo diese Substanz selbst an die Sinus grenzt, geschieht der Uebertritt der Zellen zweifellos direkt. Nun möchte ich aber daran erinnern, dass die zellarmen Partien in grösserer Breite an die Rinde herantreten als das zellreiche Parenchym und ferner haufig die Lymphbahnen, welche die Septen umschliessen, nach allen Seiten hin gleichmăssig umgeben. Bedenkt man ferner, dass die abführenden Lymphbahnen|vermutlich in der Hauptsache, wenn nicht allein, in den helleren Partien entstehen, so muss man annehmen, dass die Lymphzellen ihren Weg durch das Retikulum der zellarmeren Substanz nehmen. Und ich möchte hiermit. folgende Hypothese aufstellen: Die in den Keimzentren und dem Parenchym der zellreichen Substanz der Schweinelymphknoten gebildeten Lymphzellen wandern, soweit sie nicht direkt in die 
Vergleichende Untersuchungen über den mikroskopischen Bau etc. 497

Sinus gelangen, vom Orte ihrer Entstehung durch das Retikulum der zellarmen Substanz nach den abführenden Lymphbahnen.

Hierdurch würde auch das Fehlen der Marksubstanz in vielen Fallen, das reichliche Auftreten oberflächlicher Lymphströme verständlich und die ringförmige, an die Sinus grenzende Anordnung des helleren Teils des Parenchyms erklärlich. Die letzteren passierenden Lymphzellen treten unter dieser Annahme durch das dichtere Retikulum, teilweise unter Vermeidung der eigentlichen Lymphbahnen, ohne weiteres in die unter der Kapsel befindlichen Sinus ein.

Hund.

Wie schon in der Einleitung aus der Tabelle der Drüsen zu ersehen ist, besitzt der Hund eine geringere Zahl von Drüsenregionen als die Tiere anderer Gattungen. Ausserdem sei noch bemerkt, dass die Lymphknoten namentlich einiger Gebiete, z. B. die Drüsen des Magens, der Leber und des Halses, durchaus nicht konstant getroffen werden und bei ihrem Vorhandensein gewöhnlich nur eine geringe Grösse aufweisen. - Ich habe nun die Lymphknoten dreier Hunde unter Berücksichtigung der in der Tabelle mit * versehenen Drïsengebiete mikroskopisch untersucht und dabei folgendes gefunden.

\section{Geruistsubstanz.}

Zunächst variieren, wie bekannt, die aus Bindegewebe und etwas glatter Muskulatur bestehenden Kapseln und Septen der den verschiedenen Lymphknotengebieten angehörenden Drüsen in Bezug auf Starke und, was die Septen betrifft, auch in Bezug auf Reichlichkeit. Namentlich findet man in der Marksubstanz öfters ein etwas dichteres Netzwerk von Septen, das sich dem Hilus reichlicher zuwendet. Wenn ich nun auch beim Vergleich der einzelnen Drüsen verschiedener Regionen zwar Variationen bemerkt habe, so konnte ich doch keine regelmässige Zunahme oder Abstufung in der Stärke der Gerüstsubstanz wahrnehmen, da sich die ziemlich erheblichen Schwankungen nach meinen Präparaten fast gleichmässig auf alle Gebiete beziehen. Es liess sich nur erkennen, dass betreffs der Stärke des Gerüsts die Drüsen der beiden Körperhöhlen den äusserlich gelegenen Lymphknoten gegenüberstehen, so zwar, dass erstere im allgemeinen neben schwächerer Kapsel auch weniger Septen besitzen als 
letztere. Doch scheint mir, dass die Kniekehlendrüsen beim Hund am gerüstreichsten, Lenden-, Nieren- und Dickdarmdrüsen hingegen am gerüstärmsten sind, ohne dass man aber eine eingehendere tabellarische Ordnung vorzunehmen vermöchte.

Aehnliche Schwankungen habe ich im Auftreten wie in der Ausbildung des Hilus bemerkt, jedoch war es mir auch hier unmöglich, eine gewisse Regelmässigkeit festzustellen, die sich beispielsweise auf das Vorhandensein oder Fehlen des Hilus erstrecken könnte. Wenn v. Recklinghau sen anlässlich eines Vergleiches der Drüsen des Menschen mit denen des Hundes sagt: „Den Lymphdrüsen des Hundes fehlt gewöhnlich vollständig eine (bedeutend entwickelte) Hilussubstanz und eine entsprechend deutliche nierenförmige Gestalt. Beim Hunde tritt vielmehr die Marksubstanz mit den ausführenden Gefässen direkt an der Oberfläche des Organs zu Tage", so stehe ich, was das Fehlen der nierenförmigen Gestalt angeht, wesentlich auf demselben Standpunkte. Im übrigen sei aber folgendes hervorgehoben. Ein stark ausgeprägtes Hilusstroma sieht man schon wegen der Kleinheit verschiedener Drüsen seltener, einen $\mathrm{Hilus} \mathrm{habe}$ ich dagegen bei ungefähr $57 \%$ aller Drüsen gefunden, bei $19 \%$ war die Andeutung eines Hilus zu bemerken und nur dem Rest von $24 \%$ fehlte er. Somit dürfte obige Litteraturangabe in diesem Punkte schwerlich aufrecht zu erhalten sein. - Zu den $24 \%$ der Drüsen, welchen ein Hilus thatsächlich fehlt, gehören nach meinen Untersuchungen vor allem ausserlich gelegene Lymphknoten, während bei den in der Pleural- und Peritonealhöhle befindlichen Drüsen mit Ausnahme der Lg. lumbales und iliacae ein Hilus mindestens in schwacher Ausbildung zugegen war.

Weiterhin sei betont, dass durchaus nicht etwa bei allen Drüsen des Hundes, was wohl v. Recklinghausen auch nicht gemeint haben wird, die Marksubstanz die Oberflache erreicht, wennschon dieser Befund beim Hunde häufig zur Beobachtung gelangt. Ich habe nur bei $40 \%$ der Drüsen eine derartige oberflächliche Lagerung eines Teiles der Marksubstanz nachweisen können (cf. Fig. 8, m, o), wovon in $60 \%$ der Fälle äusserliche Drüsen, in $40 \%$ im Innern gelegene betroffen wurden. Es sei aber daran erinnert, dass die Lymphknotengebiete der erstgenannten Drüsen an sich denen der letzteren 
an Zahl überlegen sind. Trotzdem muss es auffallen, dass der höhere Prozentsatz von Lymphknoten, bei denen die Marksubstanz eine oberflächliche Lagerung erfăhrt, gemeinsam mit der Mehrzahl derjenigen Drüsen, welche keinen Hilus besitzen, den ăusserlich gelegenen Drüsenregionen zuzurechnen ist. Und so habe ich denn in vielen Fallen gefunden, dass bei den ausseren Lymphknoten das Vordringen der Marksubstanz bis an die Sinus Hand in Hand ging mit dem Fehlen des Hilus; jedoch kann ich dieses Verbăltnis nicht, wie v. Recklinghausen an einer spatteren Stelle es thut, als für alle ăusserlich gelegenen Drüsen gültig bezeichnen, da ich z. B. verschiedene äussere Drüsen gesehen habe, die neben oberflächlicher Lage des Markes einen deutlichen Hilus zeigten.

\section{Parenchym.}

Wie bereits aus dem eben Erläuterten hervorgeht, is $t$ auch die Verteilung von Rinden- und Marksubstanz bei den Drüsen desHundes eine recht verschiedene. Ich habe aber keine Drüse gesehen, die nur über eine der beiden Substanzen verfügt hätte, stets waren beide vertreten, allerdings in wechselnder Ausdehnung. Durch das Vordringen der Marksubstanz an die Oberfläche wird bei den oben genannten $40^{\circ} \circ$ der Drïsen der Zusammenhang der Rinde unterbrochen, und es werden auch bald rundliche, bald mehr langgezogene Partien derselben vom lymphoiden Rindengewebe abgetrennt. Wie schon erwähnt, gehören zu dieser Gruppe namentlich äusserlich gelegene Drüsen. Andererseits habe ich gefunden, dass bei den übrigen $60 \%$ der Lymphdrüsen eine ziemlich regelmässige Anordnung dergestalt festzustellen ist, dass die Rinde thatsächlich die Peripherie, die Marksubstanz dagegen die zentralen und die nach dem Hilus sich hinziehenden Teile der Drüse bevorzugt, dass also der Bat dieser Lymphknoten mehr oder weniger dem allgemeinen Lỵmphdrüsenschema Rechnung trägt. Diesen Befund in Wirklichkeit zu zeigen, bietcn die innerlichen Drüsen den "usseren gegenüber höhere Sicherheit, da ja bei jenen die Marksubstanz seltener Abweichungen erleidet.

\section{a. Rindensubstanz.}

Mit dem wechselnden Verhältnis der beiden lymphoiden Substanzen zu einander gehenaberauch 
Gestaltungsverschiedenheiten dieser selbsteinher. Vor allem ist die Rinde bald schmaller, bald breiter, ohne dass sich die einzelnen Drüsen nach dem Entwicklungsgrad der Rindensubstanz gruppieren lassen. Diese Thatsache kann man auch Freys Angaben in beschränktem Masse entnehmen. Frey hebt nämlich an einer Stelle seiner Spezialabhandlung die "verhältnismăssig dünne Rindenschicht der grossen Lymphknoten der Bauchhöhle" hervor und erwähnt ungefähr 20 Seiten später dasselbe von den „das Ausmaass einer Olive erreichenden Inguinaldrüsen des Hundes." Fre y hat also bei einer inneren und einer ausseren Drüse die nämliche schmale Beschaffenheit der Rindensubstanz festgestellt. Mir scheint es, als ob die Drüsen des Darmes die verhältnismässig am besten entwickelte Rindensubstanz besässen. Gestützt wird diese Meinung namentlich durch eine von mir angefertigte Schnittserie durch eine ganze Gekrösdrüise, welche in schöner gleichmassiger Ausbildung die Rindensubstanz mit ihren zahlreichen Keimzentren erkennen lässt.

Diejenigen Lymphknoten vorwiegend kleinerer Art, welche eine schmale Rindenschicht besitzen, fallen noch besonders durch folgenden Umstand auf. Infolge der gewöhnlich einschichtigen, dichten Lagerung der rundlichen oder birnförmigen Keimzentren wird nämlich die Begrenzung der Rinde gegen die Marksubstanz eine eigenartig dichtgebogte, indem die sich berührenden Keimzentren mit ihren dem Drüseninnern zugekehrten Partien bogenförmig in die Marksubstanz hineinragen, bisweilen von einem schmalen Mantel von Rindensubstanz bedeckt (cf. Fig. 8, r). Entsprechend der dichteren Zellfüllung der Keimzentren hebt sich die Rinde von der Marksubstanz in der Regel scharf ab. Meines Erachtens ist dieses bogige Eindringen der Rindensubstanz in d a s Mark eine in den Hundelymphdrüsen nicht selten auftretende Erscheinung, die ich bei etwa $22 \%$ aller Drüsen gesehen habe.

Die Keimzentren selbst fand ich von meist rundlicher Form, verschieden dicht gefüllt, teils scharf gegen die Umgebung sich abhebend, teils verschwommen, nicht selten auch aus $z$ we $\mathrm{i}$ v erschieden zellhaltigen Z onen (cf. Pferd) bestehend, in allen Drüsen des Hundes in schwankender Grösse und Zahl, entweder in einfacher Schicht oder mehrreihig über einander gelagert. Durchschnittlich zählte ich im Gesichtsfeld bei 
schwacher Vergrösserung $2,95 \mathrm{Keimzentren,} \mathrm{die} \mathrm{im}$ Mittel 0,240 mm massen. Als besonders wichtig möchte ich noch betonen, dass meine Schnitte mich zu dem Schluss berechtigen : jüngere Hunde besitzen grosse, deutliche Keimzentren, deren weniger dichte Körnung durch den Protoplasmareichtum der jungen Lymphzellen bedingt ist, auf der anderen Seite lassen ältere Tiere im allgemeinen mehr kleine, verschwommene Keimzentren erkennen.

\section{b. Marksubstanz.}

Während sich über die Mächtigkeit der Markmasse verschiedene Autoren in ihren allgemeinen Besprechungen (cf. Pferd) dahin äussern, dass in den im Innern gelegenen Lymphdrüsen die Marksubstanz in der Regel eine höhere Ausbildung darbietet als in den ausseren Lymphknoten, gestatten meine Schnitte nicht, diese Ausführungen als für die Lymphdrüsen des Hundes , in der Regel" zutreffend zu bezeichnen. Ebensowenig kann ich mich Freys speziellerer Darstellung des Verhaltens beim Hund vollkommen anschliessen, wenn er sagt: „Hier sind die grossen Lymphknoten der Bauchhöhle mit einer sehr bedeutenden Markmasse versehen, sodass die Alveolen nur eine verhältnismässig dünne Rindenschicht herstellen. Sie liegen indessen meistens in doppelter oder auch nicht selten dreifacher Reihe, meistens kleinere nach innen zeigend." Allerdings fand ich bei zwei der drei untersuchten Hunde in Schnitten durch das sogenannte Pancreas Aselli diese mehrreihige Anordnung der Keimzentren, es konnte aber infolge der hierdurch bedingten Stärke der Rindensubstanz von einem Ueberwiegen des Markes nicht gesprochen werden. Dasselbe Verhalten trat mir auch in der schon erwähnten Serie durch die Gekrösdrüse entgegen. Nur bei dem dritten Hund war die Rindenschicht der in Frage stehenden grössten Mesenterialdrüse einreihig und schwächer entwickelt als die bedeutendere Marksubstanz. Es dürften somit nicht die inneren Lymphdrüsen als die markreicheren $\mathrm{zu}$ bezeichnen sein und den äusserlich gelegenen gegenüber gestellt werden können, man muss vielmehr annehmen, dass diese beiden grossen Lymphknotengruppen sich ziemlich gleichmässig an den Schwankungen derBreitenausdehnung derMarksubstanz zu beteiligen haben.

Eine für die Marksubstanz eines Teiles der Hundelymphdrüsen charakteristische Eigenschaft ist wiederholt Gegenstand 
kurzer Besprechungen in den Abhandlungen über den histologischen Bau der Glandulae lymphaticae gewesen. Es handelt sich um das Ueberwiegen der Lymphbahnen über die Markstränge. Hierüber sagt beispielsweise v. Recklinghausen: „In den Lymphdrüsen des Hundes . . . . . nimmt die Lymphbahn relativ viel grösseren Raum ein als die follikuläre Substanz, " und Sussdorf schreibt sogar: „Beim Hunde nimmt die Lymphbahn in der Marksubstanz einen viel grösseren Raum ein als das cytogene Gewebe." Die Prüfung dieser beiden Darstellungen hat unter Bezugnahme auf meine Schnitte ergeben, dass die beiden Autoren offenbar die in einzelnen Drüsen vorgefundenen Verhältnisse ganz. allgemein auf alle Lymphknoten des Hundes übertragen haben, was kaum berechtigt sein dürfte. Es würde tiber den Rahmen dieser Arbeit hinausgehen, wollte ich meine Ansicht zahlenmåssig beweisen; denn dazu würden genaueste Messungen aller Lymphbahnen erfordertich sein. Es genüge deshalb hier die Angabe, dass ich bei ungefahr der Hälfte aller Drüsen des Hundes die Lymphbahnen der Marksubstanz breiter als die Markstränge gefunden habe (cf. Fig. 8, l), und dass dieses Verhalten nach meiner Ueberzeugung bei den Lymphknoten der Peritonealhöhle regelmässiger und schärfer zum Ausdruck gelangt als bei den übrigen Drüsen.

An den anderen in den Lymphknoten befindlichen Lmpyhwegen konnte ich nur das für die Drüsen aller Tiere gültige Schwanken in der Breite des Lumens bemerken.

Was endlich meine Untersuchungen über das Retikulum angeht, so bin ich zu demselben Ergebnis gekommen wie Frey, welcher sagt: „Ich habe die Lymphdrüsen von verschiedenem Körperstellen eines sehr grossen weiblichen Jagdhundes auf ihre Zellennetze untersucht, ohne irgendwie nennenswerten Differenzen begegnet zu sein." Auch ich habe Unterschiede im Bau des Retikulums in den verschiedenen Lymphknoten nicht finden können.

\section{Zusammenfassung.}

Die Ergebnisse meiner Untersuchungen der Lymphdrüsen sind folgende: 
Vergleichende Untersuchungen über den mikroskopischen Bau etc. 503

Pferd.

1. Die Lymphdrüsen des Pferdes lassen sich nach der Stärke der Kapsel und Septen in Gruppen ordnen, und zwar sind die dem Darmkanal und seinen Anhăngen zugehörigen Lymphdrüsen sowie die Bronchial- und Mediastinaldrüsen in der Regel gerüstarm, die sämtlichen äusserlich gelegenen Lymphknoten gerüstreich, wăhrend die den Wandungen der beiden grossen Körperhöhlen anliegenden Lymphdrüsen in der Mitte dieser zwei Gruppen stehen.

2. Das Septensystem ist vorwiegend in der Rindensubstanz schwach entwickelt.

3. Ungefähr $60 \%$ der Pferdelymphdrüsen besitzen einen Hilus, bei den übrigen $40 \%$ fehlt er.

4. Die Schwankungen im Auftreten des Hilus erstrecken sich gleichmässig auf alle Lymphdrüsengebiete.

5. Das Verhältnis der Rinden- zur Marksubstanz ist ein denkbar verschiedenes.

6. Die Rindensubstanz findet ihre höchste Entwicklung in den Bronchial-, Mediastinal- und Magenlymphdrüsen, ihre geringste dagegen in den Drüsen des Darmkanals.

7. Die Sekundarknotenbildung ist bei den gerüstreichen Lymphknoten ausgesprochener als bei den gerüstarmen. Deshalb findet man bei letzteren meist eine breit zusammenhängende Rindensubstanz.

8. In $90 \%$ aller Pferdelymphdrüsen sind Keimzentren von variierender Deutlichkeit vorhanden. Häufig treten in ihnen zwei verschieden dicht gekörnte Zonen auf.

9. Die Keimzentren liegen in der Rindensubstanz und zwar meist in der unmittelbaren Nähe der Drüsenoberfläche sowie der Septen, seltener in zentralen Partien. Letzterer Fall gelangt am häufigsten in den gerüstarmen Drüsen zur Beobachtung, die zugleich eine gering entwickelte Marksubstanz besitzen.

10. Bei schwacher Vergrösserung finden sich im Gesichtsfeld durchschnittlich 2,56 Keimzentren, die im Mittel $0,245 \mathrm{~mm}$ messen.

11. Aeltere Tiere haben weniger und undeutlichere Keimzentren als jüngere.

12. Nach der Stärke des Auftretens der Marksubstanz kann man die Lymphdrüsen in Gruppen ordnen. Am ausgepräg- 
testen ist die Marksubstanz in den Drüsen der Peritonealhöhle, am wenigsten entwickelt in den Lg. bronchiales, mediastinales und gastricae; die ausserlichen Körperlymphdrüsen stehen in der Mitte.

13. Bei $20 \%$ der Drüsen aller Regionen erreicht die Marksubstanz stellenweise die Drüsenoberfläche.

14. In manchen, namentlich äusserlich gelegenen Lymphknoten wird durch das Eindringen des hochaufsteigenden, sich weit verzweigenden Hilusstromas in die Rinde das Vorhandensein einer Marksubstanz vorgetäuscht.

15. Unterschiede im Bau des Retikulums bestehen bei den verschiedenen Drüsen nicht.

16. Die Weite der Lymphwege schwankt.

17. In $20 \%$ aller Pferdelymphdrüsen ist ausser dem bekannten System der Sinus und Lymphgänge noch ein zweites System von Kavernen und kavernösen Gängen vorhanden, welches frei das lymphoide Gewebe der Rindensubstanz durchzieht, ohne von Gerüstzügen begleitet zu sein.

\section{Rind.}

1. Kapsel und Septen sind bei den Rinderdrüsen stark entwickelt und reich an glatten Muskelfasern.

2. Die inneren Drüsen besitzen im allgemeinen eine schmälere Kapsel und weniger Septen als die ausserlichen. Gewöhnlich stehen Lenden-, Nieren-, Leber- und Darmdrüsen an der Spitze der gerüstärmeren Drüsen, während die übrigen zu den meist gerüstreichen Achsel-, Bug- und Kniekehlendrüsen den Uebergang 'bilden.

3. Die stark entwickelten und zahlreich auftretenden Trabekeln bedingen einen ausgeprägten alveolären Bau der Drüsen.

4. Ein hoch entwickeltes Septennetz zeichnet die Marksubstanz der meisten Lymphknoten aus.

5. Ein deutlicher Hilus findet sich bei etwa $70 \%$ aller Drüsen, bei $30 \%$ wird er durch eine entsprechende Kapselverstärkung ersetzt, die sich bisweilen bogenförmig in die Markmasse einbuchtet.

6. Die im Auftreten des Filus vorkommenden Unterschiede verteilen sich auf alle Regionen ungefähr gleichmässig.

7. Alle Rinderdrüsen genügen der schematischen Forderung der Verteilung von Rinden- und Marksubstanz; in manchen findet 
Vergleichende Untersuchungen über den mikroskopischen Bau etc. 505

man sogar eine Schichtung der beiden Substanzen über einander vor.

8. Rinden- und Marksubstanz sind beträchtlichen Schwankungen unterworfen, welche jedoch bei den Lymphknoten aller Gebiete ungefähr gleich stark zur Beobachtung gelangen.

9. Sämtliche Drüsen besitzen Keimzentren. Diese zeigen eine wechselnde Zellfüllung und Deutlichkeit sowie grössere Formverschiedenheiten, ohne dass hiervon einzelne Drüsen regelmässiger betroffen würden als die anderen.

10. Es finden sich im Gesichtsfeld etwa 2,38 Keimzentren mit einem durchschnittlichen Quermass von $0,276 \mathrm{~mm}$.

11. In $10 \%$ der Drüsen dringen schmale Markstreifen bis an die Oberflache vor.

12. Manche Lymphknoten lassen an den Marksträngen die Entstehung aus den Sekundarknoten und den ununterbrochenen Verlauf durch die Marksubstanz besonders schön erkennen.

13. Drüsen, bei denen Rinden- und Marksubstanz übereinander geschichtet sind und zugleich der Hilus nur durch eine Kapselverdickung angedeutet ist, besitzen ein Markgeflecht, dessen Stränge fast stets zur Kapsel parallel laufen.

14. Die Struktur der Zellennetze ist in den verschiedenen Lymphdrüsen dieselbe.

15. Die Lymphsinus zeichnen sich häufig durch besondere Breite aus.

16. Die Lymphgänge sind speziell am Uebergange der Rindenin die Marksubstanz oft besonders weit und ausgedehnt.

Schwein.

1. Die Kapsel ist im allgemeinen bei allen Schweinedrüsen schwach.

2. Das namentlich im Zentrum der Drüse gut entwickelte Septensystem geht nur in geringem Umfange Verbindungen mit der Kapsel ein.

3. Die Lymphdrüsen des Schweines lassen sich nach der Stärke der Kapsel und vor allem der Septen in Gruppen ordnen. Die Drüsen des Magens und Darmes und seiner Anhänge sind gerüstarm, die äusserlichen Drüsen gerüstreich. Die Drüsen der Brusthöhle und die übrigen Lymphknoten der Bauchhöhle schlagen zwischen beiden Gruppen die Brücke. 
4. Der Hilus ist meist nur andeutungsweise vorhanden.

5. Die Rindensubstanz bildet den überwiegenden Hauptbestandteil des ganzen Parenchyms.

6. Die Rindensubstanz besteht in allen Lymphdrüsen aus einem zellenreichen, fast stets keimzentrenhaltigen und einem zellärmeren, lymphoiden Gewebe, welches der Keimzentren entbehrt.

7. Die keimzentrenhaltige Substanz bildet in der Hauptsache mehr den Kern des Lymphknotens, den die zellärmeren Partien ringförmig umgeben und vielfach in Form breiter Streifen durchsetzen.

8. Das Retikulum der zellärmeren Substanz ist engmaschiger und in seinen einzelnen Flementen stärker gebaut als dasjenige der zellreichen Partien. Beide Retikulumarten gehen ohne Grenze in einander über.

9. Die Zellennetze der Drüsen verschiedener Regionen zeigen einen übereinstimmenden Bau.

10. Fast alle Lymphdrüsen des Schweines enthalten Keimzentren, die meist rund und scharf begrenzt sind. Bei schwacher Vergrösserung zählt man im Gesichtsfeld durchschnittlich 6,26 Keimzentren, deren Quermesser etwa 0,246 $\mathrm{mm}$ beträgt.

11. Die Marksubstanz ist sehr gering entwickelt und nur bei $30 \%$ der Drüsen nachweisbar. Sie liegt fast ausschliesslich in den periphersten Partien der helleren Substanz, aus deren Strängen sie hauptsächlich gebildet wird.

12. Der Reichtum an Keimzentren und die starke Ausdehnung. der Rindensubstanz sind charakteristische Eigenschaften der Lymphdrüsen des Schweines, durch welche sich fast alle Drüsen ohne grundsätzliche Unterschiede auszeichnen.

13. Die Lymphsinus erstrecken sich ohne bedeutende Unterbrechungen unter der Kapsel meist rund um das Parenchym, wodurch höchstwahrscheinlich oberflächliche Lymphströme in reichem Masse bedingt werden.

14. Die Lymphdrüsen des Schweines sind arm an Lymphwegen.

15. Die abführenden Lymphwege nehmen, vermutlich in der Hauptsache, ihre Entstehung in dem zellenarmeren Teile des Parenchyms.

16. Kavernenbildung ist selten. 
Vergleichende Untersuchungen über den mikroskopischen Bau etc. 507

Hund.

1. Die Kapsel und Gerüst der Lymphdrüsen des Hundes. betreffenden Schwankungen beziehen sich fast gleichmässigauf die Drüsen aller Regionen, doch besitzen die Lymphknoten der Pleural- und Peritonealhöhle im allgemeinen ein schwächeres Gerüst als die åusserlich gelegenen.

2. Ein Hilus ist bei ungefăhr $57 \%$ aller Drüsen vorhanden, bei $19 \%$ ist er angedeutet und bei $24 \%$ fehlt er. $\mathrm{Zu}$ diesen $24 \%$ gehören vor allem auusserliche Drüsen.

3. Rinden- und Marksubstanz kommen, in sehr verschiedener Ausdehnung, in allen Drüsen vor.

4. $60 \%$ der Drïsen zeigen einen regelmässigen Bau, insofern als die Rindensubstanz thatsächlich die Peripherie, die Marksubstanz dagegen die zentralen und die nach dem Hilus. hinziehenden Teile des Lymphknotens bevorzugt. Diesen Befund lassen fast regelmässig die innerlichen Drüsen erkennen.

5. Die Breite der Rindensubstanz schwankt, ohne dass sich hiernach die Drüsen gruppieren lassen.

6. Bei etwa $20 \%$ der Drüsen vorwiegend kleinerer Art, welcheeine schmale Rindenschicht besitzen, wird die Begrenzungder Rinde gegen die Marksubstanz eine eigenartig dichtgebogte dadurch, dass die rundlichen, sich berührenden Keimzentren bogenförmig in die Marksubstanz hineinragen.

7. Alle Drüsen sind durch Keimzentren ausgezeichnet, welche: meist rundlich sind, einen wechselnden Füllungsgrad haben und häufig aus zwei verschieden zellhaltigen Zonen bestehen. Durchschnittlich sind im Gesichtsfeld bei schwacher Vergrösserung 2,95 Keimzentren vorhanden, deren Quermesserungefähr $0,240 \mathrm{~mm}$ betringt.

8. Jüngere Tiere besitzen in der Regel grosse, deutliche Keimzentren, altere dagegen kleine, verschwommene.

9. Alle Lymphdrïsenregionen beteiligen sich ziemlich gleichmässigan den Schwankungen der Breitenausulehnung der Marksubstanz.

10. An $40 \%$ der Lymphknoten lässt sich eine oberflächliche Lagerung der Marksubstanz nachweisen.

11. Bei vielen äusserlichen Drüsen geht das Vordringen der Marksubstanz an die Oberfläche mit dem Fehlen des Hilus. Hand in Hand. 
12. Das Retikulum zeigt in allen Lymphdrüsen den nămlichen Bau.

13. Die Breite der Lymphbahnen schwankt unterschiedslos in allen Drüsen.

14. Ungefähr in der Hälfte der Lymphknoten aller Regionen übertreffen die Lymphwege die Markstränge an Breite.

Aus den obigen Besprechungen ergiebt sich auf die im Thema zuerst gestellte Frage die Antwort:

Es. bestehen zwischen den verschiedenen Stellen des Körpers entnommenen Lymphdrüsen von Tieren derselben Gattung histologische Unterschiede, die teils mit einer gewissen Regelmäsigkeit in Lymphknoten derselben Drüsenregion wiederkehren, teils gleichmässig a uf alle Lymphdrüsen sich erstrecken.

Wennschon in den vorangegangenen, für jede Tiergattung gesonderten Ausführungen die histologischen Unterschiede zwischen den Lymphdrüsen von Pferd, Rind, Schwein und Hund zum grossen Teil mit klargelegt worden sind, so verlangt die Beantwortung der zweiten, im Thema enthaltenen Frage „Wodurch unterscheiden sich die Lymphdrüsen der Vertreter von verschiedenen Tiergattungen (Pferd, Rind, Schwein, Hund) in Bezug auf ihren histologischen Bau?" doch noch eine kurze vergleichende Gesamtübersicht der hauptsächlichsten histologischen Unterschiede, für die natürlich die oben mitgeteilten Ergebnisse die Grundlage bilden.

Bereits in der Stärke des Stützgerüsts machen sich bedeutende Schwankungen in den Drüsen der genannten Gattungsrepräsentanten bemerkbar. Im allgemeinen besitzt das Rind die stärkste Geruistsubstanz, die zugleich am reichlichsten glatte Muskelfasern enthält, dann folgt an zweiter Stelle das Pferd, hierauf das Schwein und zum Schlusse der Hund. Bezüglich des Septensystems ist noch $z u$ erwähnen, dass dasselbe beim Rind in Rinden- und Marksubstanz gut entwickelt ist, sodass die Rinderdrüsen infolge der zahlreichen Trabekeln sowohl den alveolären Bau am besten erkennen lassen als auch das wohlentwickelste Septennetz in der Marksubstanz aufweisen; beim Hunde ist eine ähnliche Anordnung der Scheidewände vorhanden, aber nicht in so hohem Masse wie beim Rind. Anders verhalten sich Pferd 
Vergleichende Untersuchungen über den mikroskopischen Ban etc. 509

und Schwein. Die Rindensubstanz der Pferdelymphdrüsen ist in. der Regel septenarm, weshalb dieser Teil des Parenchyms häufig breit zusammenhängend auftritt, die Marksubstanz jedoch ist ziemlich septenreich; das Schwein endlich nimmt eine abweichende Stellung insofern ein, als die Trabekeln nur geringe Verbindungen mit der schwachen Kapsel eingehen, dafür aber im Innern der Drüsen um so reicher auftreten, wobei sich im Zentrum häufig ein besonders starker, gefässhaltiger Gerüstzug befindet. Aehnliche Unterschiede bestehen bezüglich des Hilus sowie des Hilusstromas. Hierbei nimmt das Schwein wieder eine Sonderstellung ein, da der Hilus höchstens durch eine Kapselverstärkung vertreten ist, also in den meisten Fallen fehlt, und somit ein eigentliches Stroma nur selten sich entwickelt hat. Auf der andern Seite steht das Rind, welches in etwa $70 \%$ aller Drüsen einen deutlichen Hilus und in den übrigen $30 \%$ wenigstens eine teils in die Marksubstanz eingebuchtete Verdickung der Kapsel zeigt. Pferd und Hund besitzen nur in ungefähr $60 \%$ der Lymphknoten einen mehr oder weniger dem Schema entsprechenden Hilus, der sonst nur angedeutet ist oder auch vielfach gänzlich fehlt. Die beste Entwicklung des Hilusstromas findet man beim Pferd und teilweise beim Rind.

In noch stärkerem Masse wie das Stützgerüst unterliegt das Parenchym Schwankungen. Das Verbältnis von Rinden- und Marksubstanz ist am konstantesten beim Rind, indem in allen Drüsen dieses Tieres die genannten Substanzen vorkommen und zwar mit wenigen Ausnahmen in regelmässiger Anordnung, sodass die Rinde meist peripher und das Mark zentral und am Hilus liegt, oder erstere bei geschichteter Lagerung den oberen, letzteres den unteren Teil der Drüse innehat. Einen ebenso konstanten, allerdings von dem der Drüsen der drei anderen Tiergattungen völlig abweichenden Bau zeigen die Drüsen des Schweines. Hier vertritt die Rindensubstanz entweder das gesamte Parenchym oder ist von einem meist unterbrochenen, peripheren Saum der gering entwickelten Marksubstanz umgeben, die sich nur selten auch im Innern der Drüse vorfindet. Grosse Unregelmässigkeiten in der Verteilung von Rinde und Mark treten dem Beobachter aber bei Pferd und Hund entgegen, was. namentlich dadurch bedingt ist, dass die Ausbreitung der Marksubstanz stark variiert und diese hierbei häufig die Rinden-- 
substanz durchdringt und an der Drüsenoberfläche zu Tage tritt. Beim Pferde kann sogar die Marksubstanz die Rinde völlig verdrăngen; auf der anderen Seite aber erfăhrt sie in einzelnen Drüsen eine so geringe Ausbildung, dass in manchen Schnitten das Parenchym nur aus Rindensubstanz besteht.

Für die Rindensubstanz sind die Keimzentren charakteristisch, die bei Rind, Schwein und Hund fast in allen Lymphknoten vorkommen, dagegen in $10 \%$ der Pferdelymphdrüsen fehlen. Die Drüsen des Schweines bedürfen noch besonderer Beachtung, weil bei ihnen ausser dem keimzentrenhaltigen Teile auch Partien der Rindensubstanz ohne Keimzentren mit Regelmässigkeit auftreten. Während beim Schwein die Keimzentren in der Mehrzahl rund und scharf gegen die Umgebung begrenzt sind, erscheinen sie bei den übrigen Tieren häufig verschwommen und erleiden auch mehrfach Abweichungen von der Kugelform. Die grössten Keimzentren besitzt das Rind, die meisten hingegen das Schwein.

Auch bezüglich des Retikulums des lymphoiden Gewebes ihrer Drüsen unterscheidet sich die zuletzt erwähnte Tiergattung von den übrigen. Obwohl auch bei Pferd, Rind und Hund die Maschen wie Gewebselemente des Zellennetzes sich nicht in allen Teilen des Parenchyms gleichen, sondern in der Marksubstanz gewöhnlich ein engeres und festeres Gefüge haben als in der Rindensubstanz, sind trotzdem den Schweinelymphdrüsen zum Unterschiede von den drei anderen Tiergattungen zwei wohl zu trennende Retikulumarten eigentümlich, wie aus dem über die Lymphknoten des Schweines handelnden Absatz zu entnehmen ist.

Betreffs der Lymphwege machen sich gleichfalls Variationen bei den verschiedenen Tiergattungen geltend. Abgesehen von physiologischen Einflïssen sind die Lymphwege, besonders die Sinus, beim Rinde am breitesten, beim Schwein am schmalsten, wie dieses Tier überhaupt eine bedeutende Armut an Lymphwegen erkennen lässt. Eine Besonderheit ungefähr der Halfte aller Hundelymphdrüsen besteht darin, dass die Lymphgänge der Marksubstanz die Stränge an Breite zum Teil bedeutend übertreffen. Und was zum Schluss das Pferd angeht, so sind etwa $20 \%$ seiner Lymphdrüsen ausser dem bekannten System der Sinus und Günge durch ein besonderes Lymphkavernensystem ausgezeichnet, welches frei das Parenchym durchsetzt. 
Vergleichende Untersuchungen über den mikroskopischen Bau etc. 511

Am Schlusse meiner Arbeit spreche ich Herrn Medicinalrat Dr. Schmorl, Prosektor am Stadtkrankenhause zu Dresden, meinen verbindlichsten Dank dafür aus, dass er mit grösster Liebenswürdigkeit die mikrophotographischen Aufnahmen meiner Retikulumpräparate gemacht hat.

\section{Litteraturangaben.}

1. G. Eckard: De glandularum lymphaticarum structura, Berolini 1858, Diss

2. F. v. Recklingha usen: Stricker, Eandbuch der Lehre von den Geweben des Menschen und der Tiere, Leipzig 1871.

3. H is : Beiträge zur Kenntnis der zum Lymphsystem gehörigen Drüsen in der Zeitschrift für Wissenschuftliche Zoologie, Bd. X, 1860 und Bd. XI. 1862.

4. L ud wig Tei c h mann: das Saugadersystem, 1861.

5. A. Kölliker: Handbuch der Gewebelehre des Menschen, 1867.

6. De rselbe: „Ueber den feineren Bau und die Funktionen der Lymphdrüsen" in den Verhandlungen der Physikalisch-Medizinischen Gesellschaft in Würzburg, 1854 .

7. H. Frey: "Untersuchungen über die Lymphdrüsen des Mensehen und der Säugetiere," Leipzig 1861.

8. Ellenberger und Ba $\mathrm{m}$ : Handbuch der Vergleichenden Anatomie der Haustiere, 1900.

9. Di es elben: Systematische und topographische Anatomie des Hundes, Berlin 1891.

10. A. B. Le e und P. M a yer: Grundzüge der mikroskopischen Technik, 1900.

11. Sussdorf: Ellenberger, Vergleichende Histologie der Haussäugetiere, Berlin 1887.

12. H. F r e y: Handbuch der Histologie und Histochemie des Menschen, 1874.

13. B r ü c ke : Sitzungsbericht und Denkschrift der Akademie der Wissenschaften zu Wien, Bd. X, 1853 bezw. 1854.

14. Philipp Stöhr: Lehrbuch der Histologie und der mikroskopischen Anatomie des Menschen, 1894.

15. W. Flemming: ,Studien über Regeneration der Gewebe; I. die Zellvermehrung in den Lymphdrüsen und verwandteu Organen und illr Einfluss auf deren Ball," im Arch. f. mikrosk. Anat., Bd. 24, 1885.

16. W. Kra a se: Handbuch der menschlichen Anatomie, I. Band (Allgemeine und mikroskopische Anatomie), 1876.

17. G. A rmauer-Hansen: Bidrag til Lymphkjertlerness normale og pathologiske Anatami," Christiania, prisbelönnet Afhandling, 1871, H. J. Jensen.

18. J. H. Chievitz: " Zur Anatomie einiger Lymphdrüsen im erwachsenen und fötalen Zustande" im Arch. f. Anat. u. Phys., Anat. Abt. 1881. 
19. A. R a u ber: Lehrbuch der Anatomie des Menschen, 1898.

20. Bill roth: Beiträge zur pathologischen Histologie, Berlin 1858.

21. Heinrich Hoyer: „Beitrag zur Kenntnis der Lymphdrüsen“ im Arch. f. mikrosk. Anat. Bd. 34. 1889.

22. Fr. Saxer: „Ueber die Entwicklung und den Bau der normalen Lymphdrüsen und die Entstehung der roten und der weissen Blutkörperchen." Anat. Hefte XIX/XX. 1896.

23. O. H e y felder: "Ueber den Bau der Lymphdrüsen," Breslan 1851.

24. Eduard Schwarz: Sitz.-Ber. der Akad. d. Wissensch. zu Wien, mathemat.-naturwiss. Klasse, Bd. 55, Abt. 1., p. 671, 1867.

25. E. Klein: Elements of Histology, London 1883.

26. Ellenberge r a nd $G$ ü $n t h$ er: Grundriss der vergleichenden Histologie der Haussäugetiere, 1901.

27. Georg Schmorl: Birch-Hirschfeld, Lehrbuch der Pathologischen. Anatomie, Leipzig 1897.

28. Donder 8: Nederl. Lancet., 3. Ser., 2. Jaarg.

\section{Erklärung der Abbildungen auf Tafel XXIV und XXV.}

Die Zeichnungen (Figur 1-8) sind von Herrn Maler Leonhardt in Leipzig angefertigt worden.

Figur 1.

Kombiniert aus einer Scham-, Achsel- und Mesenterialdrüse vom Pferd.

$$
\begin{aligned}
& K=\text { Kapsel } \\
& s=\text { Septum } \\
& h=\text { Hilus } \\
& r=\text { Rinde } \\
& m=\text { Marksubstanz, die bei } \\
& o=\text { die Oberfläche erreicht, } \\
& k z=\text { Keimzentrum mit Zone } \\
& s i=\text { Sinus. }
\end{aligned}
$$

Figur 2.

Skizze einer Leistendrüse vom Pferd.

$$
\begin{aligned}
& h s=\text { Hilusstroma } \\
& g=\text { Gefäss } \\
& r=\text { Rinde } \\
& r^{\prime}=\text { scheinbarer Markstrang. }
\end{aligned}
$$

Figur 3.

Skizze einer Milzdrüse vom Pferd.

$$
\begin{aligned}
K & =\text { Kapsel } \\
s & =\text { Septum } \\
r & =\text { Rinde } \\
c & =\text { Kaverne. }
\end{aligned}
$$


Vergleichende Untersuchungen über den mikroskopischen Bau etc. 513

\section{Figur 4.}

Skizze einer Achseldrüse vom Rind.

$$
\begin{aligned}
& K=\text { Kapsel } \\
& s=\text { Septum } \\
& h=\text { Hilus } \\
& r=\text { Rinde } \\
& m=\text { Marksubstanz } \\
& l=\text { Lymphgang. }
\end{aligned}
$$

Figur 5

Skizze einer Submaxillardrüse vom Rind.

$$
\begin{aligned}
& h=\text { Hilus } \\
& s n=\text { Septennetz } \\
& m s=\text { Markstrang } \\
& l=\text { Lymphgang. }
\end{aligned}
$$

\section{Figur 6.}

Skizze einer Magendrüse vom Rind.

$$
\begin{aligned}
& K=\text { Kapsel } \\
& s=\text { Septum } \\
& s n=\text { Septennetz } \\
& h=\text { Hilns (als Kapselverdicknng) } \\
& r=\text { Rinde } \\
& m=\text { Marksubstanz. }
\end{aligned}
$$

Figur 7.

Kombiniert aus einer Retropharyngeal- und Leberdrüse vom Schwein.

$$
\begin{aligned}
& K=\text { Kapsel } \\
& k=\text { Keimzentrum } \\
& s=\text { Septum } \\
& s^{\prime}=\text { zentrales Septum } \\
& h=\text { Hilus } \\
& r^{\cdot 1}=\text { zellenreiches Parenchym der Rindensubstanz } \\
& r^{2}=\text { zellenarmes } \\
& m=\text { Marksubstanz } \\
& s i=\text { Sinus } \\
& l=\text { Lymphgang. }
\end{aligned}
$$

Figur 8.

Skizze einer Achseldrüse vom Hund.

$$
\begin{aligned}
& r=\text { Rindensubstanz } \\
& m=\text { Marksubstanz, erreicht bei } \\
& o=\text { die Oberfläche, } \\
& l=\text { Lymphgang. }
\end{aligned}
$$

Die Figuren 9-11 sind mikrophotographische Reproduktionen von Lymphdrüsenschnitten. Sie veranschaulichen sämtlich das Stützretikulum in den Lymphdrüsen. Die rundlichen, retikulumlosen Stellen bezeichnen die Lage der Keimzentren. 
Figur 9.

Stützgerüst und Retikulum einer Mediastinaldrüse vom Rind.

Figur 10.

giebt eine Partie aus einer Milzdrüse vom Schwein bei starker Vergrösserung wieder. Sie zeigt den Uebergang der beiden Retikulumarten.

Figur 11.

Teil einer Mesenterialdrüse vom Schwein bei starker Vergrösserung.

\title{
Das Trigeminusganglion des Orang.
}

\author{
Kurze Mitteilung \\ von \\ Dr. Hermann Coenen,
}

Vol. Assistenten an der Kgl. chirurgischen Universitätsklinik zu Berlin.

Durch die Liebenswürdigkeit meines Freundes Dr. Max K o ch, Assistenten am pathologischen Institut, kam ich in die Lage, das Trigeminusganglion eines wenige Stunden vorher an Enteritis gestorbenen dreijährigen Borneo-Orangs ${ }^{1}$ ) (Simia satyrus L.) zu untersuchen. Dasselbe hat makroskopisch die grösste Aehnlichkeit mit dem des Menschen; es ist nur entsprechend kleiner. Die Fixation und Härtung geschah in $96 \%$ Alcohol, die Färbung nach Einbettung in Celloidin nach den Vorschriften von $\mathrm{N}$ is s $\mathrm{l}$. Resultate.

Die mikroskopische Tntersuchung ergab bemerkenswerte

Das die Ganglienzellen umgebende Bindegewebe ist nicht so regelmässig angeordnet, wie beim Menschen, wo jede einzelne Ganglienzelle ihren schöngeformten regelmässigen Kranz von Bindegewebszellen hat.

Die Ganglienzellen, die den Hauptvorwurf des Studiums bildeten, zeigten folgende Typen:

1. Spärlich, aber sehr in die Augen fallend sind grosse, meist hellere Ganglienzellen von runder oder etwas ovaler Form

1) Derselbe stammte aus dem Berliner Zoologischen Garten. 